\title{
Application of Fusion Rules to Classification of Subfactors
}

By

\author{
Masaki IzUmI*
}

\begin{abstract}
By the technique of Longo's sector, the following two results are obtained. (1) A proof is given to Ocneanu's announcement about the non-existence of paragroups for Coxeter graphs $E_{7}$ and $D_{\text {odd. }}$. (2) Subfactors of the type $\mathrm{III}_{1} \mathrm{AFD}$ factor with index 3 are classified.
\end{abstract}

\section{$\S 1$. Introduction and Main Results}

V. Jones theory of index [J] for subfactor of type $\mathrm{II}_{1}$ factors suddenly aroused people's interest on classification of subfactors. A. Ocneanu [O1, O2] has announced complete classification of subfactors of type $\mathrm{II}_{1}$ hyperfinite factor with index less than 4 in terms of the concept of paragroups associated with Coxeter graphs. While his announcement has not been fully published, some further analysis and classification has been obtained by S. Popa [P1, P2].

While these results are about subfactors of the type $\mathrm{II}_{1}$ factors, the notion of index by Jones has been extended to subfactors of infinite factors by $\mathrm{H}$. Kosaki [K1] and by a further work of $\mathrm{F}$. Hiai $[\mathrm{H}]$.

About 10 years before Jones' first definition of his index, S. Doplicher, $R$. Haag, and J.E. Roberts [DHR] developed the notion of statistical dimension of sectors in connection with quantum field theory or, more precisely theory of local observables. It turns out that this notion corresponds to the square root of the index of the subfactor, given by the localized morphism for the sector concerned. In recent works of $\mathrm{R}$. Longo [L1, L2] this connection between the statistical dimension and the Jones index has been clarified and a new definition of the index of subfactors of infinite factors has been given. Moreover

Communicated by H. Araki, March 8, 1991.

1991 Mathematics Subject Classification: 46 L 35

* Research Institute for Mathematical Sciences, Kyoto Univeristy, Kyoto 606, Japan. 
$\mathbb{R}$. Longo introduced the notion of sectors of an infinite factor $M$, denoted $\operatorname{Sect}(M)$, and an involutive map called conjugation in $\operatorname{Sect}(M)$, both in analogy with the case of quantum field theory. These and their properties, as described in Section 2, will be our basic tools.

Among the announcements of A. Ocneanu, one curious feature is the nonexistence of paragroup for Coxeter graphs $E_{7}$ and $D_{\text {odd }}$, one of the two main results of the present work is a proof of this non-existence result (Corollary 3.9), based on Longo's theory of $\operatorname{Sect}(M)$. While the non-existence of $E_{7}$ paragroup follows from a simple consideration on the statistical dimension of sectors, the non-existence of $D_{\text {odd }}$ paragroup follows from the fusion rules of sectors. In Section 3 we calculate fusion rules of sectors associated with Coxeter graphs $A_{n}$ and $D_{n}$. If we assume the existence of $D_{\text {odd }}$ paragroups, the calculated fusion rules are found to be inconsistent with the Perron-Frobenius eigenvectors of incidence matrix of the Coxeter graphs, thus proving the result.

The other main result of present work is a complete classification of subfactors of type $\mathbb{I I I} I_{1} \mathrm{AFD}$ factors with index 3 (Theorem 5.1). If $M$ and $N$ are type $\mathbb{I I I}_{1}$ AFD factors, $N$ being a subfactor of $M$ with index 3 , then we show in Section 5 that there exists a pair of type $\mathbb{I}_{1}$ AFD factors $R$ and $P, \mathbb{P}$ being a subfactor with index 3 and $M \supset N$ is isomorphic to $R \otimes M_{0} \supset \mathbb{P} \otimes M_{0}$ for the type $\mathbb{I I I}_{1}$ AFD factor $M_{0}$. This has been conjectured for a general case of finite index by $\mathbb{H}$. Kosaki and $\mathbb{R}$. Longo $[\mathbb{K} 2, \mathbb{K} \mathbb{L}]$. Our result implies that the classification of subfactors of the type $\mathbb{I I I}_{1}$ AFD factor with index 3 is exactly the same as the classification for the type $\mathbb{I I}_{1} \mathrm{AFD}$ factor, namely, if the principal graph is $D_{4}$ then $N$ is the fixed point algebra of $M$ under the unique outer action of $\mathbb{Z}_{3}$ and, if the principal graph is $A_{5}$ then there exist a subfactor $L$ of $N$ and an outer action of $S_{3}$ on $L$ satisfying

$$
M=L \rtimes S_{3} \supset N=L \rtimes S_{2} .
$$

As a technical tool for the above-described classification, we give in Section 4 a characterization (Theorem 4.1) of fixed point subalgebras of infinite factors under outer actions of finite groups. As a kind of generalization of this result, we give a characterization (Proposition 4.2) of depth 2.

After this work was completed, we received the paper of $Y$. Kawahigashi [Ka]. He proves the non-existence of $E_{7}$ and $D_{\text {odd }}$ paragroups and the existence of $D_{\text {even }}$ paragroups by the argument of Ocneanu's connections of graphs.

This paper is a revised version of preprint Some results on classification of subfactors. 


\section{§2. Technical Prelimimaries}

Throughout this paper we assume that von Neumann algebras have separable pre-duals and Hilbert spaces are separable. Let $M$ be a von Neumann algebra and $N$ be its von Neumann subalgebra. We denote by $\operatorname{End}(M)$ the set of unital normal endomorphisms of $M$ and by $\mathrm{E}(M, N)$ the set of faithful normal conditional expectations from $M$ to $N$. If $M$ and $N$ are factors and $E \in \mathrm{E}(M, N)$, Index $E$ denotes the Kosaki index of $E[\mathrm{~K} 1]$. If $M \supset N \supset L$ are inclusions of factors and $E_{1} \in \mathbb{E}(M, N), E_{2} \in \mathbb{E}(N, L)$, then

$$
\text { Index } E_{2} \circ E_{1}=\left(\operatorname{Index} E_{1}\right)\left(\operatorname{Index} E_{2}\right) \text {. }
$$

We denote by $[M: N]_{0}$ the minimal index of $\mathrm{F}$. Hiai $[\mathrm{H}]$, namely the minimum of Index $E$ for $E \in \mathrm{E}(M, N)$, which exists. If $\rho \in \operatorname{End}(M)$ such that $\rho(M)=$ $N$, we define the statistical dimension $d_{\rho}$ by $d_{\rho}=\left([M: N]_{0}\right)^{1 / 2}$.

2.1. Sectors and Conjugate Sectors. Almost all results quoted in this and next subsections are in $[\mathrm{L} 1, \mathrm{~L} 2]$. Let $M$ be a type III factor and $\rho_{1}, \rho_{2} \in \operatorname{End}(M)$. $\rho_{1}$ and $\rho_{2}$ are said to be unitarily equivalent if and only if there exists a unitary $u \in M$ such that

$$
\rho_{1}=\operatorname{Ad} u \circ \rho_{2} .
$$

We denote by $\operatorname{Sect}(M)$ the quotient of $\operatorname{End}(M)$ by unitary equivalence. We call elements in $\operatorname{Sect}(M)$ sectors. If $\rho \in \operatorname{End}(M)$, we denote by $[\rho]$ its class in $\operatorname{Sect}(M)$.

$\operatorname{Sect}(M)$ can be equipped with sum and product which satisfy associativity and distributivity. For given $\rho_{1}, \rho_{2} \in \operatorname{End}(M)$, we define the sum $\left[\rho_{1}\right] \oplus\left[\rho_{2}\right]$ and the product $\left[\rho_{1}\right]\left[\rho_{2}\right]$ as follows. Since we assume $M$ to be a type III factor, there exist non-zero projections $p_{1}, p_{2} \in M$ and isometries $v_{1}, v_{2} \in M$ such that

$$
p_{1}+p_{2}=1, \quad v_{i} v_{i}^{*}=p_{i}, \quad i=1,2 .
$$

We define $\rho \in \operatorname{End}(M)$ by

$$
\rho(x) \equiv v_{1} \rho_{1}(x) v_{1}^{*}+v_{2} \rho_{2}(x) v_{2}^{*}
$$

and

$$
\left[\rho_{1}\right] \oplus\left[\rho_{2}\right] \equiv[\rho], \quad\left[\rho_{1}\right]\left[\rho_{2}\right] \equiv\left[\rho_{1} \rho_{2}\right]
$$

These sum and product do not depend on the choice of $\rho_{1}, \rho_{2}$ in their classes and on the choice of $v_{1}$ and $v_{2}$. They satisfy associativity and distributivity.

We say that $\rho \in \operatorname{End}(M)$ is irreducible if and only if $M \cap \rho(M)^{\prime}=C$. If 
$\operatorname{dim} M \cap \rho(M)^{\prime}<\infty$ we can perform irreducible decomposition as follows. Let $\left\{p_{i}\right\}$ be minimal projections in $M \cap \rho(M)^{\prime}$ with $\sum_{i} p_{i}=1$ and $\left\{v_{i}\right\} \subset M$ be isometries with $v_{i} v_{i}^{*}=p_{i}$. If we define $\rho_{i} \in \operatorname{End}(M)$ by

$$
\rho_{i}(x)=v_{i}^{*} \rho(x) v_{i}, \quad x \in M,
$$

then $[\rho]=\bigoplus_{i}\left[\rho_{i}\right]$ and $\rho_{i}$ is irreducible. This decomposition does not depend on the choice of $\left\{p_{i}\right\}$ and $\left\{v_{i}\right\}$. Note that $\operatorname{dim} M \cap \rho(M)^{\prime}<\infty$ is always satisfied if $d_{\rho}<\infty$.

To define conjugate sector, we need the notion of $M-M$ correspondences $[\mathbb{P} 3]$.

A (separable) Hilbert space $H$ is called an $M-M$ correspondence if and only if $H$ is an $M-M$ bimodule and left and right actions of $M$ are $\sigma$ weakly continuous. $\quad M-M$ correspondences $H_{1}$ and $H_{2}$ are said to be equivalent if and only if there exists a surjective isometry $u: H_{1} \rightarrow H_{2}$ commuting with left and right actions. Let $H$ be an $M-M$ correspondence and $\bar{H}$ be the conjugate Hilbert space of $H$, i.e. there exists a surjective conjugate isometry from $\xi \in H$ to $\bar{\xi} \in \bar{H}$. We define the conjugate correspondence of $H$ by $\bar{H}$ in which the $M-M$ bimodule action is defined by

$$
x \cdot \bar{\xi} \circ y \equiv \overline{y^{*} \circ \xi \circ x^{*}} \quad \text { for } \quad \xi \in H, x, y \in M \text {. }
$$

On the set of unitarily equivalent classes of $M-M$ correspondences, we define the involution by the conjugate correspondence.

Let $H$ be a standard Hilbert space of $M$ and $J$ be a modular conjugation. Let $\rho \in \operatorname{End}(M)$. We define the $M-M$ correspondence $H_{\rho}$ by

$$
x \circ \xi \circ y \equiv \rho(x) J y^{*} J \xi \quad \text { for } \quad \xi \in H, \quad x, y \in M .
$$

In $\left[\mathrm{L} 2\right.$, Corollary 2.2], $\mathbb{R}$. Longo shows that $\rho \rightarrow H_{\rho}$ induces natural bijection between $\operatorname{Sect}(M)$ and the set of unitary equivalence classes of $M-M$ correspondences, and he defines conjugation in $\operatorname{Sect}(M)$ by conjugation in the latter. If $\rho \in \operatorname{End}(M)$, we denote by $[\rho]$ the conjugate sector of $[\rho]$ and, for simplicity, by $\bar{\rho}$ one of representatives of $[\bar{\rho}]$. The conjugation defined above satisfies the following relations.

$$
\begin{aligned}
\overline{\bigoplus_{i}\left[\rho_{i}\right]} & =\underset{i}{\oplus} \overline{[\rho]}, \\
\overline{\left[\rho_{1}\right]\left[\rho_{2}\right]} & =\overline{\left[\rho_{2}\right]} \overline{\left[\rho_{1}\right]}, \\
\overline{[\alpha]} & =\left[\alpha^{-1}\right] \quad \text { if } \quad \alpha \in \operatorname{Aut}(M) .
\end{aligned}
$$


We call $\rho \in \operatorname{End}(M)$ self-conjugate if and only if $[\rho]=\overline{[\rho]}$. Note that $\alpha \in$ $\operatorname{Aut}(M)$ is self-conjugate if and only if $\alpha^{2}$ is inner.

Remark. If $M$ is a type $\mathbb{I}_{\infty}$ factor, we can define $\operatorname{Sect}(M)$ in the same way, and if $\rho \in \operatorname{End}(M)$ with $d_{\rho}<\infty$, we can also perform the irreducible decomposition. To see this, it suffices to show that every non-zero projection in $M \cap$ $\rho(M)^{\prime}$ is infinite. See Appendix for the proof.

2-2. Canonical Endomorphisms and Implementations of Conditional Expectations Let $M$ and $N$ be properly infinite factors, $N$ being a subfactor of $M$, and $\Omega$ be a cyclic and separating vector simultaneously for $M$ and $N$. (Such a vector always exists). $\mathbb{R}$. Longo defined the canonical endomorphism $r_{\Omega}: M \rightarrow N$ by

$$
r_{\Omega}(x)=\Gamma_{\Omega} x \Gamma_{\Omega}^{*}, \quad x \in M
$$

where $\Gamma_{\Omega}=J_{N}^{\Omega} J_{M}^{\Omega}$ is the product of modular conjugation operators $J_{M}^{\Omega}, J_{N}^{\Omega}$ for $M$ and $N$ with respect to $\Omega$. $r_{\Omega}$ depends on $\Omega$ only up to unitaries in $N$ [L3, Theorem 1.1]. He shows in [L2, Theorem 3.1] that if $r: M \rightarrow \rho(M)$ is the canonical endomorphism for $\rho \in \operatorname{End}(M)$, then $\left.\left[\rho^{-1} \circ \gamma\right]=\overline{[\rho}\right]$. This formula gives $\bar{\rho}$ explicitly.

In [L1, Proposition 5.1], $\mathbb{R}$. Longo also shows the following important fact about implementation of conditional expectations by canonical endomorphisms. Let $M \supset N$ be a pair of properly infinite factors, $r: M \rightarrow N$ be the canonical endomorphism, and $E \in E(M, N)$. Then there exists an isometry $u \in N$ satisfying

$$
\begin{gathered}
u x=r(x) u, \quad x \in N, \\
E(x)=u^{*} r(x) u, \quad x \in M .
\end{gathered}
$$

We recall the construction of $u$. Let $\varphi$ be a faithful normal state defined by

$$
\varphi(x)=\langle E(x) \Omega, \Omega\rangle \quad x \in M .
$$

Then there is a unique vector $\xi_{0} \in L^{2}(M, \Omega)_{+}$satisfying

$$
\varphi(x)=\left\langle x \xi_{0}, \xi_{0}\right\rangle \quad x \in M .
$$

We define an isometry $u_{0} \in N^{\prime}$ as follows.

$$
u_{0} x \Omega=x \xi_{0} \quad x \in N
$$

Note that the range projection of $u_{0}$ is $e_{N}$ in the sense of Kosaki [K1, page 130]. If we define an isometry $u$ by $u=J_{N}^{\Omega} u_{0} J_{N}^{\Omega}$, then $u$ belongs to $J_{N}^{\Omega} N^{\prime} J_{N}^{\Omega}=N$ and 
satisfaies the above conditions.

If $M$ is isomorphic to $N$ and $\rho \in \operatorname{End}(M)$ with $\rho(M)=N$, then there exists an isometry $v \in M$ satisfying

$$
v x=\bar{\rho} \rho(x) v, \quad E(x)=\rho\left(v^{*} \bar{\rho}(x) v\right), \quad x \in M .
$$

Let $\phi$ be a normal, unital, completely positive map on $M$. We can construct a natural $M-M$ correspondence as follows. Let $H$ be a standard Hilbert space of $M$ and $\Omega$ be a cyclic and separatig vector for $M$. Then $\Omega$ is a cyclic vector for the opposite algebra $M^{0}=J M J$, i.e.

$$
\overline{\Omega M}=\overline{J M J \Omega}=H \text {. }
$$

We define positive semi-definite sesquilinear form on $M \otimes_{\mathrm{alg}} M$ by

$$
\left(\sum_{i} x_{i} \otimes y_{i}, \sum_{j} u_{j} \otimes v_{j}\right) \equiv \sum_{i, j}\left\langle\phi\left(u_{j}^{*} x_{i}\right) \Omega y_{i}, \Omega v_{j}\right\rangle \text {. }
$$

Like G.N.S. construction, we can construct an $M-M$ correspondence out of this sesquilinear form and, by using the bijection between $\operatorname{Sect}(M)$ and correspondences, we obtain $\rho_{\phi} \in \operatorname{End}(M)$ and $\xi \in \mathbb{H}$ satisfying

$$
\left\langle\rho_{\phi}(x) \xi y, \xi\right\rangle=\langle\phi(x) \Omega y, \Omega\rangle, \quad \overline{\rho_{\phi}(M) \xi M}=H .
$$

If $\phi=E$ and $\Omega$ is a cyclic and separating vector for $M$ and $N$, then

$$
\left\langle\rho_{\phi}(x) \xi y, \xi\right\rangle=\langle E(x) \Omega y, \Omega\rangle=\left\langle u^{*} \gamma(x) u \Omega y, \Omega\right\rangle=\langle\gamma(x) u \Omega y, u \Omega\rangle .
$$

So, if $\overline{\gamma(M) u \Omega M}=H$, we obtain $\left[\rho_{E}\right]=[r]$. We prove $\overline{\gamma(M) u \Omega M}=H$ as follows. Let $p$ be the projection onto $\overline{\gamma(M) u \Omega M^{\perp}}$. Then $p$ belongs to $M \cap r(M)^{\prime}$ and $p u \Omega=0$. Since $\Omega$ is a separating vector for $M, p u=0$ and $p u u^{*}=p J_{N}^{\Omega} e_{N} J_{N}^{\Omega}$ $=0$. So $0=J_{N}^{\Omega} p J_{N}^{\Omega} e_{N} \xi_{0}=J_{N}^{\Omega} p J_{N}^{\Omega} \xi_{0}$. Due to $p \in M \cap \gamma(M)^{\prime}$

$$
J_{N}^{\Omega} p J_{N}^{\Omega} \in J_{N}^{\Omega} M J_{N}^{\Omega} \cap J_{M}^{\Omega} M^{\prime} J_{M}^{\Omega}=J_{N}^{\Omega} M J_{N}^{\Omega} \cap M .
$$

Since $\xi_{0}$ is a separating vector for $M$, we obtain $p=0$ and so $\overline{r(M) u \Omega M}=H$.

Moreover if $M$ is isomorphic to $N$ and $\rho \in \mathbb{E n d}(M)$ with $\rho(M)=N$, then $\left[\rho_{E}\right]=[\gamma]=[\rho \bar{\rho}]$. In Sections 4 and 5 , we calculate $[\rho \bar{\rho}]$ from the conditional expectation by the above formula.

2.3. Minimal Expectations and the Basic Construction. Let $M$ be a properly infinite factor and $\rho \in \mathbb{E n d}(M)$ with $d_{\rho}<\infty$. The tower associated with $M \supset$ $\rho(M)$ is [L2, Corollary 4.14]

$$
M \supset \rho(M) \supset \rho \bar{\rho}(M) \supset \rho \bar{\rho} \rho(M) \supset \cdots \text { 。 }
$$


This formula enable us to analyze the index theory by sectors. By decomposing $\left[(\rho \bar{\rho})^{n}\right]$ and $\left[(\rho \bar{\rho})^{n} \rho\right]$, we get new sectors, which we call descendant sectors.

To calculate statistical dimensions of descendant sectors, we use Hiai's minimal expectation [H].

If $M \supset N$ are a pair of factors with a finite index and $E \in \mathrm{E}(M, N)$, the following are equivalent $[\mathrm{H}][\mathrm{L} 1]$ :

(i) $E$ is the minimal expectation.

(ii) $\left.E\right|_{M \cap N^{\prime}}$ and $\left.E^{-1}\right|_{M \cap N^{\prime}}$ are traces and $\left.E^{-1}\right|_{M \cap N^{\prime}}=$ (Index $\left.E\right)\left.E\right|_{M \cap N^{\prime}}$. ( $E^{-1}$ is a map from $N^{\prime}$ to $M^{\prime}$, canonically defined from $E[\mathrm{C}, \mathrm{Ha}]$.)

(iii) Index $E_{e}=E(e)^{2}$ (index $E$ ) for all projections $e \in N^{\prime} \cap M$ where $E_{e}=$ $\left.E(e)^{-1} E(\cdot) e\right|_{M_{e}}$.

By (iii), if $\left\{e_{i}\right\}$ is a family of mutually orthogonal projections in $M \cap N^{\prime}$ with $\sum_{i} e_{i}=1$, then

$$
\sum_{i}\left(\operatorname{Index} E_{e_{i}}\right)^{1 / 2}=(\operatorname{Index} E)^{1 / 2} \sum_{i} E\left(e_{i}\right)=(\operatorname{Index} E)^{1 / 2} .
$$

This means that if $[\rho]=\bigoplus_{i}\left[\rho_{i}\right]$, then $d_{\rho}=\sum_{i} d_{\rho_{i}}$ for $\rho, \rho_{i} \in \operatorname{End}(M)$. This is the reason why we use $d_{\rho}$ instead of the index.

$\mathbb{H}$. Kosaki and $\mathbb{R}$. Longo show the mutiplicativity of the minimal index in case of the basic construction [KL], and using this, $R$. Longo shows the multiplicativity in general case [L4]. Let $M \supset N \supset L$ be inclusions of factors with finite indices and $E_{1} \in \mathrm{E}(M, N), E_{2} \in \mathrm{E}(N, L)$ be the minimal expectations. Then $E_{1} \circ E_{2}$ is also the minimal expectation from $M$ to $L$ and so $[M: N]_{0}[N: L]_{0}$ $=[M: L]_{0}$ by (2.1). This means that if $\rho_{1}, \rho_{2} \in \operatorname{End}(M)$ with $d_{\rho_{1}}, d_{\rho_{2}}<\infty$, then $d_{\rho_{1} \rho_{2}}=d_{\rho_{3}} d_{\rho_{2}}$.

Let

$$
M=N_{-1} \supset N_{0} \supset N_{1} \supset N_{2} \cdots
$$

be the tower associated with the inclusion of factors $M \supset N_{0}$, and $\varepsilon_{i} \in \mathrm{E}\left(N_{i-1}, N_{i}\right)$ be the minimal expectations. If we define $E_{n}$ by

$$
E_{n}=\varepsilon_{n} \circ \varepsilon_{n-1} \circ \cdots \circ \varepsilon_{0},
$$

then $E_{n}$ turns out to be the minimal expectation from $M$ to $N_{n}$, and $\left[M: N_{n}\right]_{0}=$ $\left[M: N_{0}\right]_{0}^{n+1}$.

Since $E_{n}$ is a trace on $M \cap N_{n}^{\prime}$ by (ii),

$$
\left.\varphi \equiv \lim _{n} E_{n}\right|_{\underset{m}{\vee} M \cap N_{m}^{\prime}}
$$

is a trace on $\underset{m}{\bigvee} M \cap N_{m}^{\prime}$. Moreover, if depth of $M \supset N_{0}$ is finite, it is well-known 
that $\varphi$ is the unique trace and values of $\varphi$ on minimal projections of $M \cap N_{n}^{\prime}$ are determined by the Perron-Frobenius eigenvector of the principal graph. [GHJ]

Let $\rho \in \operatorname{End}(M)$ with $\rho(M)=N$ and $\left[(\rho \bar{\rho})^{m}\right]=\bigoplus_{i}\left[\rho_{i}\right]$ be the irreducible decomposition, with $\left\{p_{i}\right\}$ the corresponding minimal projections in $M \cap$ $(\rho \bar{\rho})^{m}(M)^{\prime}$. Then

$$
d_{\rho_{i}}=\left(\operatorname{Index}\left(E_{2 m-1}\right)_{\rho_{i}}\right)^{1 / 2}=E_{2 m-1}\left(p_{i}\right)\left(\operatorname{Index} E_{2 m-1}\right)^{1 / 2}=\varphi\left(p_{i}\right)\left(d_{\rho}\right)^{2 m} .
$$

A similar calculation holds for $\left[(\rho \bar{\rho})^{m} \rho\right]$. So we can calculate $d_{\rho_{i}}$ from the Perron-Frobenius eigenvector.

2.4. Characterization of conjugate sectors The most important tool of this paper is the following theorem.

Theorem 2.1. Let $M$ be a properly infinite factor and $\rho_{1}, \rho_{2} \in \operatorname{End}(M)$ with $d_{\rho_{1}}, d_{\rho_{2}}<\infty$. If $\rho_{1}$ and $\rho_{2}$ are irreducible, then the following conditions are equivalent:

(i) $\left[\rho_{1}\right]\left[\rho_{2}\right]$ or $\left[\rho_{2}\right]\left[\rho_{1}\right]$ contains the identity sector $[i d]$.

(ii) $\left.\left[\rho_{1}\right]=\overline{\left[\rho_{2}\right.}\right]$.

If $\rho_{1}$ and $\rho_{2}$ satisfy the above conditions, $\left[\rho_{1}\right]\left[\rho_{2}\right]$ and $\left[\rho_{2}\right]\left[\rho_{1}\right]$ contain $[i d]$ with multiplicity one.

This theorem is essentially due to $\mathbb{R}$. Longo [L2, Theorem 4.1]. The only improvement over his theorem is in the condition (i), in which we demand that one of $\left[\rho_{1}\right]\left[\rho_{2}\right]$ and $\left[\rho_{2}\right]\left[\rho_{1}\right]$ contains $[i d]$, instead of both. For the proof of (i) $\Rightarrow$ (ii), we may assume that $\left[\rho_{1}\right]\left[\rho_{2}\right]$ contains $[i d]$ and also assume that $d_{\rho_{1}} \leqq d_{\rho_{2}}$ (if not, we can consider $\left[\overline{\rho_{2}}\right]\left[\overline{\rho_{1}}\right]$ instead). Then his proof for (i) $\Rightarrow$ (ii) applies to our case.

Before closing this section, we would like to pose the following question.

Question 2.2. Let $M$ be a properly infinite factor and $N$ be a given subfactor of $M$ which is isomorphic to $M$. Can we find a self-conjugate $\rho \in \operatorname{End}(M)$ such that $\rho(\mathbb{M})=N$ ?

In general, the answer is no. We will show an affirmative case in Section 3 and a negative case in Section 4.

\section{§3。 Funsion $\mathbb{R} u$ ules of Sectors}

Let $M \supset N$ be a pair of factors with $[M: N]<4$. Then it is well-known 
that the principal graph is one of the Coxeter graphs $A_{n}, D_{n}, E_{6}, E_{7}, E_{8}$ [GHJ, Corollary 4.6.6]. Moreover, A. Ocneanu states in [O1, page 162] that $D_{\text {odd }}$ and $E_{7}$ do not appear, but no complete proof has been published until now. In this section, we compute the fusion rules of sectors, which is the rules of the irreducible decomposition of sectors, associated with the above graphs and prove the non-appearance of $D_{\text {odd }}$ and $E_{7}$.

Let $M$ be a properly infinite factor and $\rho_{1} \in \operatorname{End}(M)$ with $d_{\rho_{1}}<\infty$. Since

$$
M \supset \rho_{1}(M) \supset \rho_{1} \bar{\rho}_{1}(M) \supset \rho_{1} \bar{\rho}_{1} \rho_{1}(M) \supset \cdots
$$

is the tower associated with $M \supset \rho_{1}(M)$ as quoted in Subsection 2.3, the Bratteli diagram of

$$
M \cap M^{\prime}=\mathbb{C} \subset M \cap \rho_{1}(M)^{\prime} \subset M \cap \rho_{1} \bar{\rho}_{1}(M)^{\prime} \subset M \cap \rho_{1} \bar{\rho}_{1} \rho_{1}(M)^{\prime} \subset \cdots
$$

is decided by the principal graph of $M \supset \rho_{1}(M)$. We saw in Subsection 2.3 that minimal projections in $M \cap\left(\rho_{1} \bar{\rho}_{1}\right)^{n}(M)^{\prime}$ and $M \cap\left(\rho_{1} \bar{\rho}_{1}\right)^{n} \rho_{1}(M)^{\prime}$ correspond to descendant sectors. This means that the Bratteli diagram gives fusion rules of descendant sectors of $\rho_{1}$. We show this more precisely.

Let $\rho, \sigma \in \operatorname{End}(M)$ and $[\rho]=\bigoplus_{i \in I} m(i)\left[\rho_{i}\right],\left[\rho_{i}\right][\sigma]=\bigoplus_{j \in J} n(i, j)\left[\sigma_{j}\right]$ be the irreducible decompositions of $[\rho]$ and $\left[\rho_{i}\right][\sigma]$, where $m(i)$ and $n(i, j)$ are the multiplicities and $n(i, j)$ may take 0 . Then there are isometries $\left\{S(i)_{k}\right\}_{i \in I, k=1,2, \cdots, m_{i}}$ $\left\{T(i, j)_{l}\right\}_{i \in I, j \in J, l=1,2, \cdots, n(i, j)}$ in $M$ satisfaying the following.

$$
\begin{aligned}
& \sum_{i \in I} \sum_{k=1}^{m(i)} S(i)_{k} S(i)_{k}^{*}=1, \\
& \sum_{j \in J}^{n} \sum_{l=1}^{n(i, j)} T(i, j)_{l} T(i, j)_{l}^{*}=1 \\
& \rho(x)=\sum_{i \in I} \sum_{k=1}^{m(i)} S(i)_{k} \rho_{i}(x) S(i)_{k}^{*}, \\
& \rho_{i} \circ \sigma(x)=\sum_{j \in J} \sum_{l=1}^{n(i, j)} T(i, j)_{l} \sigma_{j}(x) T(i, j)_{l}^{*}, \\
& \rho \circ \sigma(x)=\sum_{j \in J}\left[\sum_{i \in I} \sum_{k=1}^{m(i)} \sum_{l=1}^{n(i, j)} S(i)_{k} T(i, j)_{l} \sigma_{j}(x) T(i, j)_{l}^{*} S(i)_{l}^{*}\right] .
\end{aligned}
$$

Due to (3.0.3) and (3.0.5), the central decompositions of $M \cap \rho(M)^{\prime}$ and $M \cap$ $\rho \circ \sigma(M)^{\prime}$ are as follows.

$$
\begin{aligned}
& M \cap \rho(M)^{\prime}=\bigoplus_{i \in I} A(i), \\
& M \cap \rho \circ \sigma(M)^{\prime}=\bigoplus_{j \in J} B(j), \\
& A(i) \equiv C^{*}\left(S(i)_{k_{1}} S(i)_{k_{2}}^{*}\right)_{k_{1}, k_{2}}, \\
& B(j) \equiv C^{*}\left(S\left(i_{1}\right)_{k_{1}} T\left(i_{1}, j\right)_{l_{1}} T\left(i_{2}, j\right)_{l_{2}}^{*} S\left(i_{2}\right)_{k_{2}}^{*}\right)_{i_{1}, i_{2}, k_{1}, k_{2}, l_{1}, l_{2}} .
\end{aligned}
$$


Note that $\left\{S(i)_{k_{1}} S(i)_{k_{2}}^{*_{2}}\right\}_{k_{1}, k_{2}}$ and $\left\{S\left(i_{1}\right)_{k_{1}} T\left(i_{1}, j\right)_{l_{1}} T\left(i_{2}, j\right)_{l_{2}}^{*_{2}} S\left(i_{2}\right)_{k_{2}}^{*}\right\}_{i_{1}, i_{2}, k_{1}, k_{2}, l_{1}, l_{2}}$ are the matrix units of $A(i)$ and $B(j)$. Thanks to (3.0.2), a minimal projection $S(i)_{k} S(i)_{k}^{*}$ in $A(i)$ is also written as follows.

$$
S(i)_{k} S(i)_{k}^{*}=\sum_{j \in J}\left[\sum_{l=1}^{n(i, j)} S(i)_{k} T(i, j)_{l} T(i, j)_{l}^{*} S(i)_{k}^{*}\right] \text {. }
$$

This means that in the Bratteli diagram of $\bigoplus_{i \in I} A(i) \subset \bigoplus_{j \in J} B(j)$, there are $n(i, j)$ edges between $A(i)$ and $B(j)$, where $n(i, j)$ is the multiplicity of $\left[\sigma_{j}\right]$ in $\left[\rho_{i}\right][\sigma]$.

We assume that the principal graph is one of $A_{n}, D_{n}, E_{6}, E_{7}, E_{8}$ and explain the details of computations one by one.

3.1. The Case of $A_{2 n}$ For $A_{n}$, the corresponding subfactors are constructed in [J, Thoerem 4.3.2]. The Bratteli diagram is as in Fig. 1.

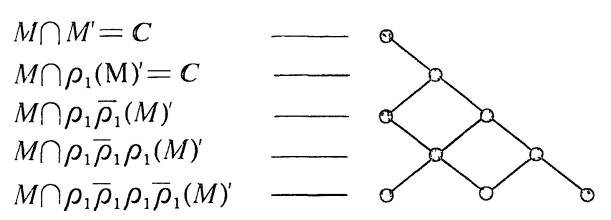

Fig. 1. The Bratteli diagram in the case of $A_{2 n}$.

First we look at the level of $M \cap \rho_{1} \bar{\rho}_{1}(M)^{\prime}=\mathbb{C} \oplus \mathbb{C}$. Since there are two points in this level, $\left[\rho_{1} \bar{\rho}_{1}\right]$ is decomposed into two sectors. One of these is [id] by the characterization of the conjugate sector in Subsection 2.4 and call the other sector as $\left[\rho_{2}\right]$. The next question is which of two points corresponds to [id]. The answer is the left one. Indeed, since

$$
\left[\rho_{1} \bar{\rho}_{1} \rho_{1}\right]=\left[\rho_{1} \bar{\rho}_{1}\right]\left[\rho_{1}\right]=\left([i d] \oplus\left[\rho_{2}\right]\right)\left[\rho_{1}\right]=[i d]\left[\rho_{1}\right] \oplus\left[\rho_{2}\right]\left[\rho_{1}\right]
$$

we see from the diagram that one of $[i d]\left[\rho_{1}\right]$ and $\left[\rho_{2}\right]\left[\rho_{1}\right]$ is decomposed into two sectors. But $[i d]\left[\rho_{1}\right]=\left[\rho_{1}\right]$ is irreducible and then the left one corresponds to [id].

Next we look at the level of $M \cap \rho_{1} \bar{\rho}_{1} \rho_{1}(M)^{\prime} \simeq M_{2} \oplus \mathbb{C} . \quad$ By the above argument, we know that $\left[\rho_{1} \bar{\rho}_{1} \rho_{1}\right]$ contains $\left[\rho_{1}\right]$ twice and hence $\left[\rho_{2}\right]\left[\rho_{1}\right]$ contains $\left[\rho_{1}\right]$. We call the other irreducible component of $\left[\rho_{2}\right]\left[\rho_{1}\right]$ as $\left[\rho_{3}\right]$. By repeating such an argument, we get Fig. 2, where each sector is mutually different in each row, and the following basic fusion rules. $\left(\left[\rho_{0}\right]=[i d]\right.$ in $k=0$ equation. $)$

$$
\begin{aligned}
{\left[\rho_{2 k+1}\right]\left[\bar{\rho}_{1}\right] } & =\left[\rho_{2 k}\right] \oplus\left[\rho_{2 k+2}\right], \quad k=0,1, \cdots, n-2, \\
{\left[\rho_{2 n-1}\right]\left[\bar{\rho}_{1}\right] } & =\left[\rho_{2 n-2}\right], \\
{\left[\rho_{2 k}\right]\left[\rho_{1}\right] } & =\left[\rho_{2 k-1}\right] \oplus\left[\rho_{2 k+1}\right], \quad k=1,2, \cdots, n-1 .
\end{aligned}
$$




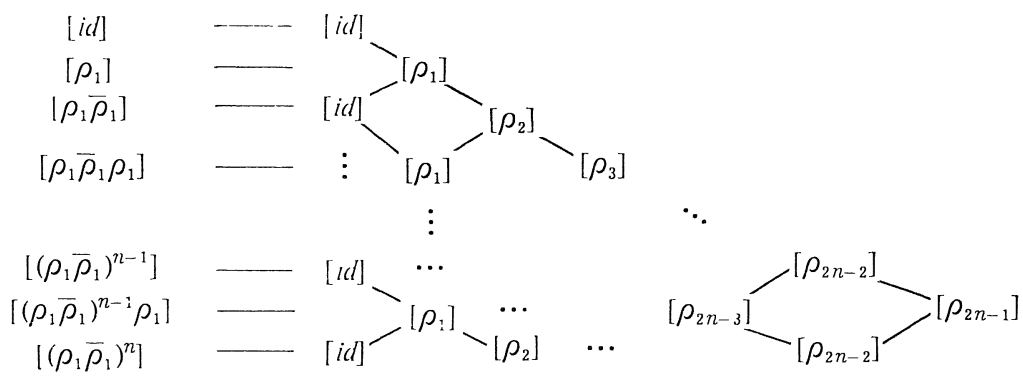

Fig. 2. The diagram of fusion rules in the case of $A_{2 n}$.

We can also compute the products of other pairs:

\section{Lemma 3.1.}

$$
\begin{aligned}
{\left[\bar{\rho}_{2 k+1}\right]\left[\rho_{2}\right] } & =\left[\bar{\rho}_{2 k-1}\right] \oplus\left[\bar{\rho}_{2 k+1}\right] \oplus\left[\bar{\rho}_{2 k+3}\right], \quad k=1,2, \cdots, n-2, \\
{\left[\bar{\rho}_{1}\right]\left[\rho_{2}\right] } & =\left[\bar{\rho}_{1}\right] \oplus\left[\bar{\rho}_{3}\right], \\
{\left[\bar{\rho}_{2 n-1}\right]\left[\rho_{2}\right] } & =\left[\bar{\rho}_{2 n-3}\right], \\
{\left[\rho_{2 k}\right]\left[\rho_{2}\right] } & =\left[\rho_{2 k-2}\right] \oplus\left[\rho_{2 k}\right] \oplus\left[\rho_{2 k+2}\right], \quad k=1,2, \cdots, n-2, \\
{\left[\rho_{2 n-2}\right]\left[\rho_{2}\right] } & =\left[\rho_{2 n-4}\right] \oplus\left[\rho_{2 n-2}\right] .
\end{aligned}
$$

Proof. Since $\left[\rho_{1} \bar{\rho}_{1}\right]=[i d] \oplus\left[\rho_{2}\right]$, we may compute $\left[\bar{\rho}_{2 k+1}\right]\left[\rho_{1} \bar{\rho}_{1}\right]$ and $\left[\rho_{2 k}\right]$ $\left[\rho_{1} \bar{\rho}_{1}\right]$ instead of the above products because of the uniqueness of the irreducible decomposition. $\left[\rho_{2 k}\right]\left[\rho_{1} \bar{\rho}_{1}\right]$ is easily obtained by going down from the level $\left(\rho_{1} \bar{\rho}_{1}\right)^{k}$ to the level $\left(\rho_{1} \bar{\rho}_{1}\right)^{k+1}$ (namely by 2 levels), starting from $\left[\rho_{2 k}\right]$. To compute $\left[\bar{\rho}_{2 k+1}\right]\left[\rho_{1} \bar{\rho}_{1}\right]$ we use fusion rules of descendant sectors of $\left[\bar{\rho}_{1}\right]$. It is easy to see that if $d_{\rho_{1}}<2$ the principal graphs of $M \supset \rho_{1}(M)$ and $M \supset \bar{\rho}_{1}(M)$ coincide because $M \supset \bar{\rho}_{1}(M)$ is isomorphic to $M_{1} \supset M$ where $M_{1}$ is the extension of $M$ by $\rho_{1}(M)$ [L2, page 296]. (Or more directly, due to $\overline{\left[\left(\rho_{1} \bar{\rho}_{1}\right)^{n} \rho_{1}\right]}=\left[\left(\bar{\rho}_{1} \rho_{1}\right)^{n} \bar{\rho}_{1}\right], \operatorname{dim} Z$ $\left(M \cap\left(\rho_{1} \bar{\rho}_{1}\right)^{n} \rho_{1}(M)^{\prime}\right)=\operatorname{dim} Z\left(M \cap\left(\bar{\rho}_{1} \rho_{1}\right)^{n} \bar{\rho}_{1}(M)^{\prime}\right)$ and so the principal graph of $M \supset \bar{\rho}_{1}(M)$, which is one of $A_{n}, D_{n}, E_{6}, E_{7}$, and $E_{8}$, must coincide with that of $\left.M \supset \rho_{1}(M)\right)$. Let $\left\{\left[\hat{\rho}_{i}\right]\right\}_{i=1}^{2 n-1} \subset \operatorname{Sect}(M)$ be the descendant sectors of $\left[\bar{\rho}_{1}\right]$. Since $\overline{\left[\left(\bar{\rho}_{1} \rho_{1}\right)^{k} \bar{\rho}_{1}\right]}=\left[\left(\rho_{1} \bar{\rho}_{1}\right)^{k} \rho_{1}\right]$, we get $\left[\hat{\rho}_{2 k+1}\right]=\left[\bar{\rho}_{2 k+1}\right]$. And we can compute $\left[\bar{\rho}_{2 k+1}\right]\left[\rho_{1} \bar{\rho}_{1}\right]$ from the diagram of $\left\{\hat{\rho}_{i}\right\}_{i=1}^{2 n-1}$.

Q.E.D.

The Perron-Frobenius eigenvector $\left(v_{j}\right)_{j=1}^{2 n}$ of $A_{2 n}$ is given by $v_{j}=\left(\sin \frac{j \pi}{2 n+1}\right) /$ $\left(\sin \frac{\pi}{2 n+1}\right)$ (see Fig. 3). 


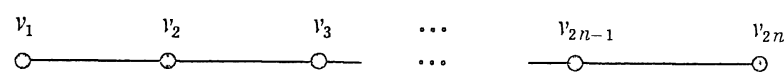

Fig. 3. The Perron-Frobenius eigenvector of $A_{2 n}$.

As we saw in Subsection 2.3, we can compute $d_{\rho_{i}}$ from the PerronFrobenius eigenvector:

$$
d_{\rho_{i}}=\frac{\sin \frac{i+1}{2 n+1} \pi}{\sin \frac{\pi}{2 n+1}} .
$$

Note that $d_{\rho_{2 n-1}}=1$. This means $\rho_{2 n-1} \in \operatorname{Aut}(M)$ and we write $\alpha=\rho_{2 n-1}$ If $\theta_{1}$ and $\theta_{2}$ in $\operatorname{End}(M)$ have the same image $\theta_{1}(M)=\theta_{2}(M)$, there is a $\beta \in \operatorname{Aut}(M)$ such that $\theta_{1}=\theta_{2} \circ \beta$. In other word, if we analyze subfactors by endomorphisms, we have the ambiguity of multiplying automorphisms from right in the choice of the endomorphism representing the subfactor. In the following proposition, we see that we can use this ambiguity to cancel $\alpha$ to make all $\left[\rho_{k}\right]$ self-conjugate. Thus the answer to the Question 2.2 in Section 2 is yes in this case.

Proposition 3.2. In the case of $A_{2 n}$, a self-conjugate $\rho_{1}$ can be chosen such that $\left[\rho_{k}\right]=\left[\rho_{2 n-1-k}\right]$ and all descendant sectors $\left[\rho_{k}\right]$ are self-conjugate. The diagram of fusion rules is as in Fig. 4.

Proof. We go back to Fig. 2. It is easy to see that $\left[\rho_{2 k}\right]$ is self-conjugate by induction because $[i d]$ and $\left[\left(\rho_{1} \bar{\rho}_{1}\right)^{k}\right]$ are self-conjugate. Since $[\alpha]\left[\bar{\rho}_{1}\right]=\left[\rho_{2 n-1}\right]$ $\left[\bar{\rho}_{1}\right]=\left[\rho_{2 n-2}\right]$ by $(3.1 .2)$, we get $\left[\rho_{1}\right]\left[\alpha^{-1}\right]=\left[\rho_{2 n-2}\right]$ by conjugation. We now show $\left[\rho_{2 k-1}\right]=\left[\rho_{2 n-2 k}\right][\alpha]$ or equivalently $\left[\bar{\rho}_{2 k-1}\right]=\left[\alpha^{-1}\right]\left[\rho_{2 n-2 k}\right]$ for $k=1,2, \cdots, n$. For $k=1$ we know $\left[\bar{\rho}_{1}\right]=\left[\alpha^{-1}\right]\left[\rho_{2 n-2}\right]$ by the above argument. By using Lemma 3.1 and induction, we obtain the desired equality.

Due to $\left[\rho_{1}\right]\left[\alpha^{-1}\right]=\left[\rho_{2 n-2}\right]$, we have $\rho_{2 n-2}(M)=\rho_{1}(M)$ so that we can take $\rho_{2 n-2}$ instead of $\rho_{1}$. Then $\left[\rho_{1}\right]$ is replaced by $\left[\rho_{1}\right]\left[\alpha^{-1}\right]=\left[\rho_{1} \alpha^{-1}\right]$ and hence

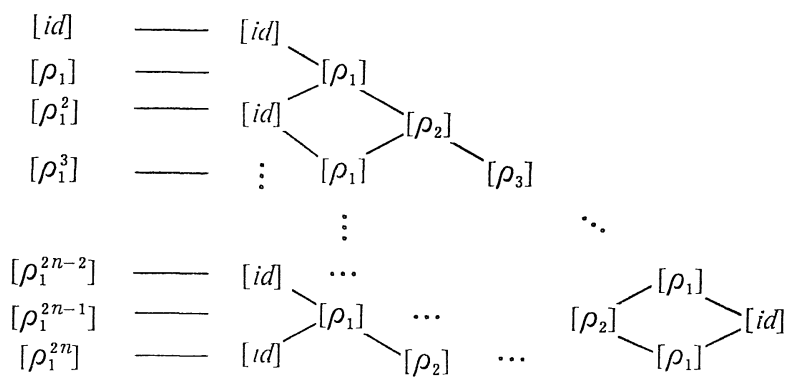

Fig. 4. The diagram of fusion rules in Proposition 3.2. 
$\left[\left(\rho_{1} \bar{\rho}_{1}\right)^{k} \rho_{1}\right]$ turns into $\left[\left(\rho_{1} \bar{\rho}_{1}\right)^{k} \rho_{1}\right]\left[\alpha^{-1}\right]$, while $\left[\left(\rho_{1} \bar{\rho}_{1}\right)^{k}\right]$ does not change. So we complete the proof.

Q.E.D.

In principle, we can compute every principal graph of $M \supset \rho_{i}(M)$ from fusion rules, because

$$
M \supset \rho_{i}(M) \supset \rho_{i} \bar{\rho}_{i}(M) \supset \rho_{i} \bar{\rho}_{i} \rho_{i}(M) \supset \cdots
$$

is the associated tower. For example, due to (3.1.7) and (3.1.8) that of $M \supset$ $\rho_{2}(M)$ is as in Fig. 5.

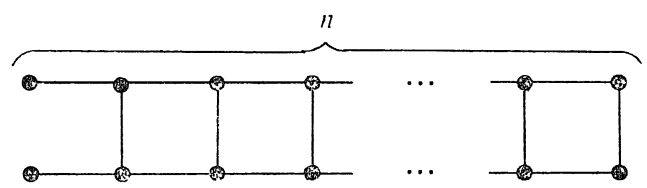

Fig. 5. The principal graph of $M \supset \rho_{2}(M)$ in the case of $A_{2 n}$.

3.2. The Case of $A_{2 n+1}$ The diagram of fusion rules is as in Fig. 6 and the basic fusion rules and the statistical dimensions of descendant sectors are as follows.

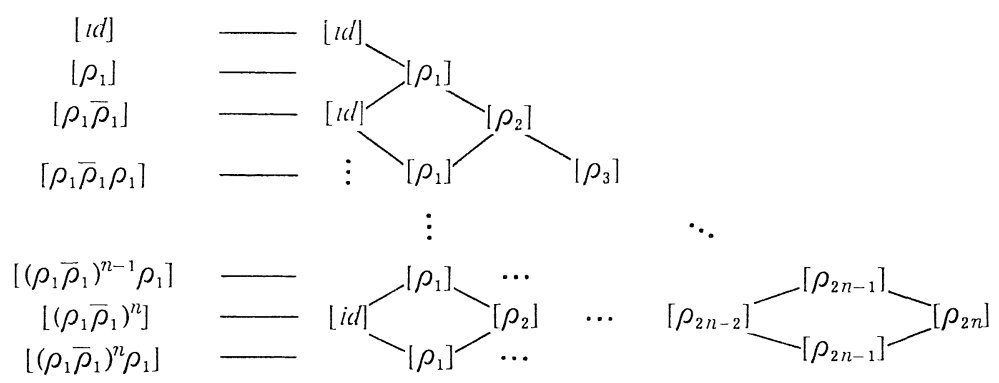

Fig. 6. The diagram of fusion rules in the case of $A_{2 n+1}$.

$$
\begin{aligned}
{\left[\rho_{2 k+1}\right]\left[\bar{\rho}_{1}\right] } & =\left[\rho_{2 k}\right] \oplus\left[\rho_{2 k+2}\right] \quad k=0,1, \cdots, n-1 \\
{\left[\rho_{2 k}\right]\left[\rho_{1}\right] } & =\left[\rho_{2 k-1}\right] \oplus\left[\rho_{2 k+1}\right] \quad k=1,2, \cdots, n-1 \\
{\left[\rho_{2 n}\right]\left[\rho_{1}\right] } & =\left[\rho_{2 n-1}\right]
\end{aligned}
$$

$$
d_{\rho_{i}}=\frac{\sin \frac{i+1}{2 n+2} \pi}{\sin \frac{\pi}{2 n+2}} .
$$

As in Subsection 3.1, $\rho_{2 n} \in \operatorname{Aut}(M)$ and we write $\alpha=\rho_{2 n}$. Difference from 
the case of $A_{2 n}$ is that $\alpha$ is self-conjugate and an invariant of the subfactor $\rho_{1}(M) .[\alpha]$ plays essential rôle in Theorem 5.1.

Proposition $\mathbb{H}_{0}$ 3。 (i) $\left[\rho_{2 n-k}\right]=[\alpha]\left[\rho_{k}\right]$ and especially $\left[\rho_{n}\right]=[\alpha]\left[\rho_{n}\right]$.

(ii) We can take representatives $\alpha$ and $\rho_{n}$ such that $\alpha$ is a $\mathbb{Z}_{2}$ action and $\rho_{n}(M) \subset M^{\alpha}$ where $M^{\alpha}$ is the fixed point algebra under $\alpha$.

Proof. (i): This is easily shown by induction. (ii): Since $\left[\rho_{n}\right]=[\alpha]\left[\rho_{n}\right]$, we can take a representative $\alpha$ such that $\rho_{n}=\alpha \circ \rho_{n}$. As we mentioned at the end of Subsection 2.1, $\alpha^{2}$ is inner and $\alpha^{2} \circ \rho_{n}=\rho_{n}$. Since $\rho_{n}$ is irreducible so $M \cap \rho_{n}(M)^{\prime}=\mathbb{C}$ and then $\alpha^{2}=i d . \quad \rho_{n}(M) \subset M^{\alpha}$ follows from $\alpha \circ \rho_{n}=\rho_{n}$.

Q.E.D.

For later use, we show the following lemma.

Lemma 3.48. If $M$ is the type III $I_{1}$ AFD factor, then we can take $\rho_{1}$ satisfying $\left[\rho_{1}\right][\alpha]=[\alpha]\left[\rho_{1}\right]$.

Proof. By the work of Y. Kawahigashi, C.E. Surtherland and M. Takesaki [KST, Theorem 20], an outer action of $\mathbb{Z}_{2}$ on the type $\mathbb{H}_{1} \mathrm{AFD}$ factor is unique as a cocycle conjugacy class. As in the proof of Lemma 3.1, if $\left\{\left[\hat{\rho}_{i}\right]\right\}_{i=1}^{2 n}$ are descendant sectors of $\bar{\rho}_{1}$ then $\left[\hat{\rho}_{2 i+1}\right]=\left[\bar{\rho}_{2 i+1}\right]$ and we may assume that $\hat{\rho}_{2 n}$ is a $\mathbb{Z}_{2}$ action. So there exists a $\theta \in \operatorname{Aut}(M)$ such that

$$
\left[\hat{\rho}_{2 n}\right]=[\theta][\alpha]\left[\theta^{-1}\right] \text {. }
$$

Since $\left[\rho_{2 n-1}\right]=[\alpha]\left[\rho_{1}\right]$ and $\left[\bar{\rho}_{2 n-1}\right]=\left[\hat{\rho}_{2 n}\right]\left[\bar{\rho}_{1}\right]$ from Lemma 3.3, we have

$$
[\alpha]\left[\rho_{1}\right]=\overline{\left[\hat{\rho}_{2 n}\right]\left[\bar{\rho}_{1}\right]}=\left[\rho_{1}\right]\left[\hat{\rho}_{2 n}\right]=\left[\rho_{1}\right][\theta][\alpha]\left[\theta^{-1}\right]
$$

and $[\alpha]\left[\rho_{1} \circ \theta\right]=\left[\rho_{1} \circ \theta\right][\alpha]$. We can take $\rho_{1} \circ \theta$ instead of $\rho_{1}$. Then $\left[\rho_{2 k}\right]$ are unchanged and in particular $[\alpha]$ remains the same. So we have $\left[\rho_{1}\right][\alpha]=[\alpha]\left[\rho_{1}\right]$ for new $\rho_{1}$.

Q.E.D.

The principal graph of $M \supset \rho_{2}(M)$ is as in Fig. 7 .

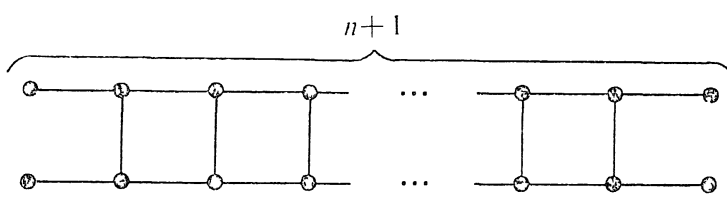

Fig. 7. The principal graph of $M \supset \rho_{2}(M)$ in the case of $A_{2 n+1}$.

In [W1], subfactors with index $\left(\frac{\sin (k / l) \pi}{\sin \pi / l}\right)^{2}(l=3,4, \cdots, k=1,2, \cdots)$ appear 
twice, once in page 360 and once in page 380. Note that the former for $k=3$ and our $M \supset \rho_{i}(M)$ have the same principal graphs. Since the graphs in Fig. 5 and Fig. 7 do not coincide with the principal graphs of the latter [W2], for $k=3$ the above two subfactors are different. The author would like to thank Y. Kawahigashi who suggested the author the above difference and showed him the note of Wenzl's lecture at the Mittag-Leffler Institute in 1988.

3.3. The Case of $E_{6}, E_{7}$, and $E_{8}$. Suppose that the principal graph of $M \supset$ $\rho_{1}(M)$ is $E_{7}$. The descendant sectors are as in Fig. 8 where we write only the principal part.

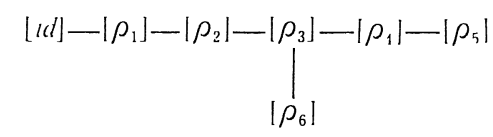

Fig. 8. The descendant sectors in the case of $E_{7}$.

We can compute $d_{p_{i}}$ from the Perron-Frobenius eigenvector as before and we obtain

$$
d_{\rho_{5}}=\frac{\sin \frac{6}{18} \pi}{4 \cos ^{2} \frac{\pi}{18} \sin \frac{\pi}{18}}=2 \cos \frac{5}{18} \pi<2 \cos \frac{\pi}{4} .
$$

This value is not admissible as a statistical dimension [J, Theorem 4.3.1] $[\mathrm{K} 1$, Theorem 5.4] and hence we have a contradiction.

If we take $E_{6}$ and $E_{8}$, we can confirm that there is no problem. Suppose that the principal graph of $M \supset \rho_{1}(M)$ is $E_{6}$. The descendant sectors are as in Fig. 9.

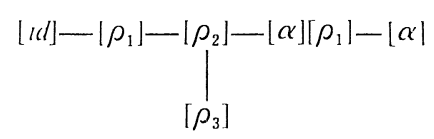

Fig. 9. The descendant sectors in the case of $E_{6}$.

The statistical dimensions are as follows.

$$
d_{\rho_{1}}=2 \cos \frac{\pi}{12}, d_{\rho_{2}}=\frac{\sin \frac{3}{12} \pi}{\sin \frac{\pi}{12}}, d_{\rho_{3}}=2 \cos \frac{\pi}{4}, d_{\alpha}=1 .
$$

As in the case of $A_{2 n+1}, \alpha$ is a $Z_{2}$ action. This is a reflection of the $\boldsymbol{Z}_{2}$ symmetry of bicolored labelled $E_{6}$. Fusion rules of descendant sectors are as follows. 


$$
\begin{aligned}
{\left[\rho_{1} \bar{\rho}_{1}\right] } & =[i d] \oplus\left[\rho_{2}\right], \\
{\left[\rho_{2} \rho_{1}\right] } & =\left[\rho_{1}\right] \oplus\left[\rho_{3}\right] \oplus\left[\alpha \rho_{1}\right], \\
{\left[\rho_{2}\right] } & =\left[\rho_{3} \bar{\rho}_{1}\right]=\left[\rho_{1} \bar{\rho}_{3}\right], \\
{\left[\rho_{2}\right]^{2} } & =[i d] \oplus[\alpha] \oplus 2\left[\rho_{2}\right], \\
{\left[\rho_{2} \rho_{3}\right] } & =\left[\rho_{1}\right] \oplus\left[\alpha \rho_{1}\right], \\
{\left[\rho_{3} \bar{\rho}_{3}\right] } & =[i d] \oplus[\alpha] .
\end{aligned}
$$

(3.3.3) shows the existence of the following inclusions of factors.

$$
\begin{gathered}
M \supset \rho_{1}(M) \supset \rho_{2}(M), \\
{\left[\rho_{1}(M): \rho_{2}(M)\right]_{0}=\left[M: \bar{\rho}_{3}(M)\right]_{0}=2 .}
\end{gathered}
$$

These inclusions suggest the way to construct an inclusion with the principal graph $E_{6}$. In [I], we construct $\rho_{2}$ on the type $\mathrm{III}_{1 /(1+v \overline{3})}$ AFD factor by using Cuntz algebra $O_{4}$. From $\left[\rho_{1}(M): \rho_{2}(M)\right]_{0}=2$, the relation between $\rho_{1}(M)$ and $\rho_{2}(M)$ is the crossed product by a $\mathbb{Z}_{2}$ action. Using this fact, we also construct an inclusion of factors with the principal graph $E_{6}$. The principal graph of $M \supset \rho_{2}(M)$ is as in Fig. 10.

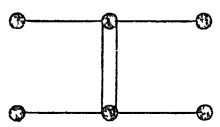

Fig. 10. The principal graph of $M \supset \rho(M)_{2}$ in the case of $E_{6}$.

Suppose that the principal graph of $M \supset \rho_{1}(M)$ is $E_{8}$. The descendant sectors are as in Fig. 11.

$$
\mid i c]-\left[\rho_{1}\right]-\left[\rho_{2}\right]-\left[\rho_{3}\right]-\left[\rho_{1}\right]-\left[\rho_{5}\right]-\left[\rho_{6}\right]
$$

Fig. 11. The descendant sectors in the case of $E_{8}$.

The minimal statistical dimension of the descendant sectors is given by

$$
\min \left\{d_{\rho_{i}} ; i=1,2, \cdots, 7\right\}=d_{\rho_{6}}=2 \cos \frac{\pi}{5} .
$$

Note that $d_{p_{i}}>2$ for $i \neq 1,6$. By computing fusion rules of descendant sectors, we have the following relations.

$$
\left[\rho_{3}\right]=\left[\rho_{6} \rho_{7}\right],
$$




$$
\begin{aligned}
& {\left[\rho_{4}\right]=\left[\rho_{6} \rho_{2}\right]=\left[\rho_{7} \bar{\rho}_{1}\right],} \\
& {\left[\rho_{5}\right]=\left[\rho_{6} \rho_{1}\right] .}
\end{aligned}
$$

From $d_{\rho_{6}}=2 \cos \frac{\pi}{5}$, the principal graph of $M \supset \rho_{6}(M)$ is $A_{4}$. In analogy with the case of $E_{6}$, it is possible to say " $E_{8}$ has $A_{4}$ symmetry". The principal graph of $M \supset \rho_{2}(M)$ is as in Fig. 12.

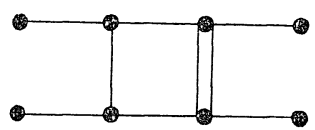

Fig. 12. The principal graph of $M \supset \rho_{2}(M)$ in the case of $E_{8}$.

3.4. The Case of $D_{2 n+3}$. Suppose that the principal graph of $M \supset \rho_{1}(M)$ is $D_{2 n+3}, n \geq 1$. The descendant sectors are as in Fig. 13 .

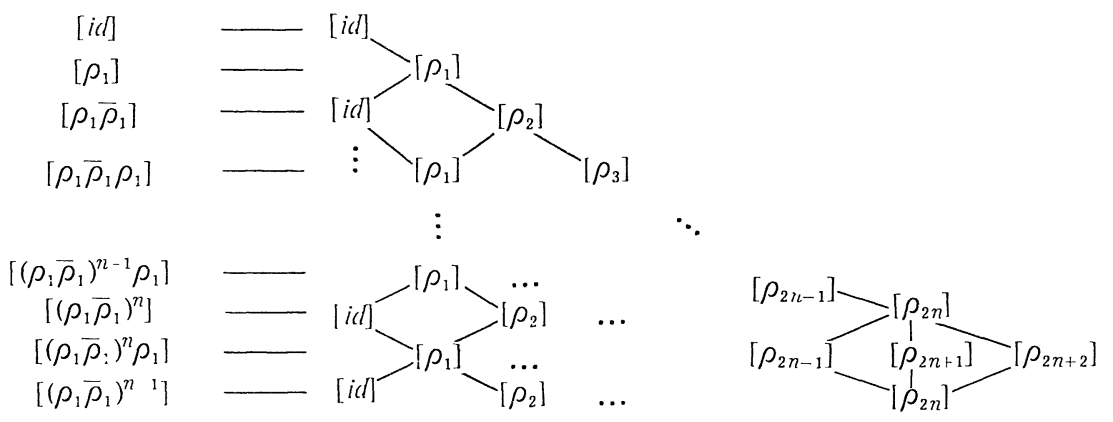

Fig. 13. The diagram of fusion rules in the case of $D_{2 n+3}$.

The basic fusion rules are given by

$$
\begin{aligned}
{\left[\rho_{2 k+1}\right]\left[\bar{\rho}_{1}\right] } & =\left[\rho_{2 k}\right] \oplus\left[\rho_{2 k+2}\right], \quad k=0,1, \cdots, n-1, \\
{\left[\rho_{2 n+1}\right]\left[\bar{\rho}_{1}\right] } & =\left[\rho_{2 n+2}\right]\left[\bar{\rho}_{1}\right]=\left[\rho_{2 n}\right], \\
{\left[\rho_{2 k}\right]\left[\rho_{1}\right] } & =\left[\rho_{2 k-1}\right] \oplus\left[\rho_{2 k+1}\right], \quad k=1,2, \cdots, n-1, \\
{\left[\rho_{2 n}\right]\left[\rho_{1}\right] } & =\left[\rho_{2 n-1}\right] \oplus\left[\rho_{2 n+1}\right] \oplus\left[\rho_{2 n+2}\right] .
\end{aligned}
$$

The statistical dimensions are given by

$$
d_{\rho_{i}}=\frac{\sin \frac{i+1}{4 n+4} \pi}{\sin \frac{\pi}{4 n+4}}, \quad i=0,1, \cdots, 2 n,
$$




$$
d_{\rho_{2 n+1}}=d_{\rho_{2 n+2}}=\frac{1}{2 \sin \frac{\pi}{4 n+4}}
$$

We note that $\left[\rho_{i}\right], i=0,1, \cdots, 2 n+1,2 n+2$ must be mutually different, for example due to the difference values of their statistical dimensions. $\left[\rho_{2 n+1}\right]$ and $\left[\rho_{2 n+2}\right]$ also must be different because if not, the principal graph changes.

\section{Lemma 3.5。}

$$
d_{\rho_{2 n+1} \bar{\rho}_{2 n+1}}=d_{\rho_{2_{n+2}} \bar{\rho}_{2_{n+2}}}=d_{\rho_{2_{n+1}} \bar{\rho}_{2 n+2}}=d_{\rho_{2 n+2} \bar{\rho}_{2 n+1}}=\frac{1}{4 \sin ^{2} \frac{\pi}{4 n+4}} .
$$

Proof. Due to $d_{\rho_{2 n+1}}=d_{\rho_{2 n+2}}=\frac{1}{2 \sin \frac{\pi}{4 n+4}}$ and the multiplicativity of the statistical dimension, the equalities hold.

Lemma 3.6. (i)

$$
\begin{aligned}
{\left[\bar{\rho}_{2 k+1}\right]\left[\rho_{2}\right] } & =\left[\bar{\rho}_{2 k-1}\right] \oplus\left[\bar{\rho}_{2 k+1}\right] \oplus\left[\bar{\rho}_{2 k+3}\right], \quad k=1,2, \cdots, n-2, \\
{\left[\bar{\rho}_{1}\right]\left[\rho_{2}\right] } & =\left[\bar{\rho}_{1}\right] \oplus\left[\bar{\rho}_{3}\right], \\
{\left[\bar{\rho}_{2 n-1}\right]\left[\rho_{2}\right] } & =\left[\bar{\rho}_{2 n-3}\right] \oplus\left[\bar{\rho}_{2 n-1}\right] \oplus\left[\bar{\rho}_{2 n+1}\right] \oplus\left[\bar{\rho}_{2 n+2}\right], \\
{\left[\bar{\rho}_{2 n+1}\right]\left[\rho_{2}\right] } & =\left[\bar{\rho}_{2 n-1}\right] \oplus\left[\bar{\rho}_{2 n+2}\right], \\
{\left[\bar{\rho}_{2 n+2}\right]\left[\rho_{2}\right] } & =\left[\bar{\rho}_{2 n-1}\right] \oplus\left[\bar{\rho}_{2 n+1}\right], \\
{\left[\rho_{2 k}\right]\left[\rho_{2}\right] } & =\left[\rho_{2 k-2}\right] \oplus\left[\rho_{2 k}\right] \oplus\left[\rho_{2 k+2}\right], \quad k=1,2, \cdots, n-1, \\
{\left[\rho_{2 n}\right]\left[\rho_{2}\right] } & =\left[\rho_{2 n-2}\right] \oplus 2\left[\rho_{2 n}\right] .
\end{aligned}
$$

(ii)

$$
\begin{aligned}
& {\left[\rho_{2 n+1}\right]\left[\bar{\rho}_{2 k+1}\right] }=\left[\rho_{2 n+2}\right]\left[\bar{\rho}_{2 k+1}\right] \\
&=\left[\rho_{2(n-k)}\right] \oplus\left[\rho_{2(n-k+1)}\right] \oplus \cdots \oplus\left[\rho_{2 n}\right], k=0,1, \cdots, n-1, \\
& {\left[\rho_{2 n+1}\right]\left[\bar{\rho}_{2 n+1}\right] \oplus\left[\rho_{2 n+1}\right]\left[\bar{\rho}_{2 n+2}\right]=[i d] \oplus\left[\rho_{2}\right] \oplus \cdots \oplus\left[\rho_{2 n}\right] . }
\end{aligned}
$$

(iii)

$$
\begin{aligned}
& {\left[\rho_{2 n+1}\right]\left[\bar{\rho}_{2 n+1}\right]\left[\rho_{2}\right]=\left[\rho_{2}\right] \oplus\left[\rho_{4}\right] \oplus \cdots\left[\rho_{2 n}\right] \oplus\left[\rho_{2 n+1}\right]\left[\bar{\rho}_{2 n+2}\right],} \\
& {\left[\rho_{2 n+1}\right]\left[\bar{\rho}_{2 n+2}\right]\left[\rho_{2}\right]=\left[\rho_{2}\right] \oplus\left[\rho_{4}\right] \oplus \cdots\left[\rho_{2 n}\right] \oplus\left[\rho_{2 n+1}\right]\left[\bar{\rho}_{2 n+1}\right] .}
\end{aligned}
$$

Proof. (i): This can be obtained in the same way as the proof of Lemma 3.1.

(ii): For $k=0$, we already have $\left[\rho_{2 n+1}\right]\left[\bar{\rho}_{1}\right]=\left[\rho_{2 n+2}\right]\left[\bar{\rho}_{1}\right]=\left[\rho_{2 n}\right]$. For $k=1$, the 
equality holds from $\left[\rho_{2 n+1}\right]\left[\bar{\rho}_{1}\right]\left[\rho_{2}\right]=\left[\rho_{2 n+2}\right]\left[\bar{\rho}_{1}\right]\left[\rho_{2}\right]=\left[\rho_{2 n}\right]\left[\rho_{2}\right]$ and (i). In the same way, general case holds by induction.

(iii): From (i) and (ii), we obtain

$$
\begin{aligned}
{\left[\rho_{2 n+1}\right]\left[\bar{\rho}_{2 n+1}\right]\left[\rho_{2}\right] } & =\left[\rho_{2 n+1}\right]\left(\left[\bar{\rho}_{2 n-1}\right] \oplus\left[\bar{\rho}_{2 n+2}\right]\right) \\
& =\left[\rho_{2}\right] \oplus\left[\rho_{4}\right] \oplus \cdots \oplus\left[\rho_{2 n}\right] \oplus\left[\rho_{2 n+1}\right]\left[\bar{\rho}_{2 n+2}\right]
\end{aligned}
$$

Second equality is shown in the same way.

Q.E.D.

Theorem 3.7. Let $M \supset N$ be a pair of properly infinite factors with $M \simeq N$ and $[M: N]<\infty$. Then the principal graph of $M \supset N$ is neither $E_{7}$ nor $D_{\text {odd }}$.

Proof. In subsection 3.3, we have seen that $E_{7}$ can not appear. Suppose that the principal graph of $M \supset N=\rho_{1}(M)$ is $D_{2 n+3}$. In (3.4.13), [id], [ $\left.\rho_{2}\right], \cdots,\left[\rho_{2 n}\right]$ divide into two groups. By characterization of the conjugate sector in Subsection 2.4, $\left[\rho_{2 n+1}\right]\left[\bar{\rho}_{2 n+1}\right]$ contains $[i d]$. So, the right hand side of $(3.4 .15)$ contains $[i d]$. Then, $\left[\rho_{2 n+1}\right]\left[\bar{\rho}_{2 n+2}\right]$ on the left hand side must contain $\left[\bar{\rho}_{2}\right]=\left[\rho_{2}\right]$.

We now investigate the balance of the $\left[\rho_{2}\right]$ term in (3.4.14). On the right hand side, $\left[\rho_{2}\right]$ appears twice, once as $\left[\rho_{2}\right]$ and once in $\left[\rho_{2 n+1}\right]\left[\bar{\rho}_{2 n+2}\right]$ as we have seen. On the left hand side, $\left[\rho_{2 n+1}\right]\left[\bar{\rho}_{2 n+1}\right]$ is a partial sum of the terms on the right hand side of (3.4.13), among which only $[i d],\left[\rho_{2}\right]$ and $\left[\rho_{4}\right]$ can produce $\left[\rho_{2}\right]$ after being multiplied by $\left[\rho_{2}\right]$ from right. Since $\left[\rho_{2}\right]$ is in $\left[\rho_{2 n+1}\right]\left[\bar{\rho}_{2 n+2}\right]$, it can not be in $\left[\rho_{2 n+1}\right]\left[\bar{\rho}_{2 n+1}\right]$. Therefore $\left[\rho_{2 n+1}\right]\left[\bar{\rho}_{2 n+1}\right]$ must contain $\left[\rho_{4}\right]$.

By repeating the same type of arguments, we get the following equations. The case of odd $n$ :

$$
\begin{aligned}
& {\left[\rho_{2 n+1}\right]\left[\bar{\rho}_{2 n+1}\right]=[i d] \oplus\left[\rho_{4}\right] \oplus \cdots \oplus\left[\rho_{2 n-2}\right],} \\
& {\left[\rho_{2 n+1}\right]\left[\bar{\rho}_{2 n+2}\right]=\left[\rho_{2}\right] \oplus\left[\rho_{6}\right] \oplus \cdots \oplus\left[\rho_{2 n}\right] .}
\end{aligned}
$$

The case of even $n$ :

$$
\begin{aligned}
& {\left[\rho_{2 n+1}\right]\left[\bar{\rho}_{2 n+1}\right]=[i d] \oplus\left[\rho_{4}\right] \oplus \cdots \oplus\left[\rho_{2 n}\right],} \\
& {\left[\rho_{2 n+1}\right]\left[\bar{\rho}_{2 n+2}\right]=\left[\rho_{2}\right] \oplus \cdots \oplus\left[\rho_{2 n-2}\right] .}
\end{aligned}
$$

By Lemma 3.5, $d_{\rho_{2 n+1} \bar{\rho}_{2 n+1}}=d_{\rho_{2 n+1} \bar{\rho}_{2 n+2}}$. But this is impossible because of $d_{\rho_{i}}=$ $\frac{\sin ((i+1) /(4 n+4)) \pi}{\sin (\pi /(4 n+4))} i=1,2, \cdots, 2 n$ and the additivity of the statistical dimensions. (Note that $d_{\rho_{k}}<d_{\rho_{l}}$ if $k<l \leq 2 n$.)

Q.E.D.

To obtain the result in general case, we need the following lemma.

Lemma 3.8. (R. Longo [L4, Lemma 2.3]) Let $M \supset N$ be a pair of factors. 
Then there exists a factor $L$ such that $M \otimes L$ is isomrophic to $N \otimes L$.

Corollary 3.9. Let $M \supset N$ be a pair of any factors with $[M: N]<\infty$. Then the principal graph of $M \supset N$ is neither $E_{7}$ nor $D_{\text {odd }}$.

Proof. Since the principal graph dose not change after taking tensor product, we may assume that $M$ and $N$ are properly infinite and $M$ is isomrophic to $N$ due to Lemma 3.8. Then Theorem 3.7 applies.

Q.E.D.

3.5 The Case of $D_{2 n+2}$. Suppose that the principal graph of $M \supset \rho_{1}(M)$ is $D_{2 n+2}$. The diagram of fusion rules is as in Fig. 14 and the basic fusion rules and the statistical dimensions of the descendant sectors are as follows.

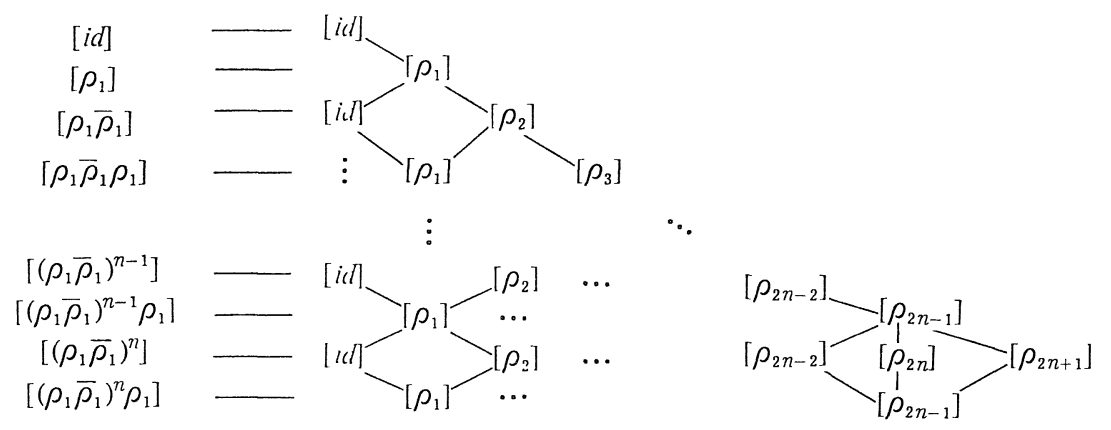

Fig. 14. The diagram of fusion rules in the case of $D_{2 n+2}$.

$$
\begin{aligned}
{\left[\rho_{2 k+1}\right]\left[\bar{\rho}_{1}\right] } & =\left[\rho_{2 k}\right] \oplus\left[\rho_{2 k+2}\right], \quad k=0,1, \cdots, n-2, \\
{\left[\rho_{2 n-1}\right]\left[\bar{\rho}_{1}\right] } & =\left[\rho_{2 n-2}\right] \oplus\left[\rho_{2 n}\right] \oplus\left[\rho_{2 n+1}\right], \\
{\left[\rho_{2 k}\right]\left[\rho_{1}\right] } & =\left[\rho_{2 k-1}\right] \oplus\left[\rho_{2 k+1}\right], \quad k=1,2, \cdots, n-1, \\
{\left[\rho_{2 n}\right]\left[\rho_{1}\right] } & =\left[\rho_{2 n+1}\right]\left[\rho_{1}\right]=\left[\rho_{2 n-1}\right] . \\
d_{\rho_{i}} & =\frac{\sin \frac{i+1}{4 n+2} \pi}{\sin \frac{\pi}{4 n+2} \quad i=1,2, \cdots, 2 n-1,} \\
d_{\rho_{2 n}} & =d_{\rho_{2 n+1}} \\
= & \frac{1}{2 \sin \frac{\pi}{4 n+2}} \begin{cases}=1 & n=1 \\
=2 \cos \frac{\pi}{5} & n=2 \\
>2 & n>2 .\end{cases}
\end{aligned}
$$

By a similar argument as in the proof of Lemma 3.6, we get the following fusion rules 


$$
\begin{gathered}
{\left[\rho_{2 n}\right]\left[\rho_{2 k}\right]=\left[\rho_{2 n-2 k}\right] \oplus\left[\rho_{2 n-2 k+2}\right] \oplus \cdots \oplus\left[\rho_{2 n-2}\right] \begin{cases}\oplus\left[\rho_{2 n+1}\right] & \text { for odd } k, \\
\oplus\left[\rho_{2 n}\right] & \text { for even } k,\end{cases} } \\
{\left[\rho_{2 n+1}\right]\left[\rho_{2 k}\right]=\left[\rho_{2 n-2 k}\right] \oplus\left[\rho_{2 n-2 k+2}\right] \oplus \cdots \oplus\left[\rho_{2 n-2}\right] \begin{cases}\oplus\left[\rho_{2 n}\right] & \text { for odd } k, \\
\oplus\left[\rho_{2 n+1}\right] & \text { for even } k,\end{cases} } \\
\text { for } 1 \leqq k \leqq n-1 .
\end{gathered}
$$

$\left[\rho_{2 n}\right]\left[\rho_{2 n}\right] \oplus\left[\rho_{2 n}\right]\left[\rho_{2 n+1}\right]=[i d] \oplus\left[\rho_{2}\right] \oplus \cdots \oplus\left[\rho_{2 n-2}\right] \begin{cases}\oplus\left[\rho_{2 n+1}\right] & \text { for odd } n, \\ \oplus\left[\rho_{2 n}\right] & \text { for even } n,\end{cases}$

$\left[\rho_{2 n+1}\right]\left[\rho_{2 n}\right] \oplus\left[\rho_{2 n+1}\right]\left[\rho_{2 n+1}\right]=[i d] \oplus\left[\rho_{2}\right] \oplus \cdots \oplus\left[\rho_{2 n-2}\right] \begin{cases}\oplus\left[\rho_{2 n}\right] & \text { for odd } n, \\ \oplus\left[\rho_{2 n+1}\right] & \text { for even } n\end{cases}$

Proposition 3.10. If $n$ is odd, then $\left[\rho_{2 n+1}\right]$ is the conjugate sector of $\left[\rho_{2 n}\right]$ and the following fusion rules hold.

$$
\begin{aligned}
{\left[\rho_{2 n}\right]\left[\rho_{2 n}\right] } & =\left[\rho_{2}\right] \oplus\left[\rho_{6}\right] \oplus \cdots\left[\rho_{2 n-4}\right] \oplus\left[\rho_{2 n+1}\right], \\
{\left[\rho_{2 n}\right]\left[\rho_{2 n+1}\right] } & =[i d] \oplus\left[\rho_{4}\right] \oplus \cdots\left[\rho_{2 n-6}\right] \oplus\left[\rho_{2 n-2}\right] .
\end{aligned}
$$

If $n$ is even, then $\left[\rho_{2 n}\right]$ and $\left[\rho_{2 n+1}\right]$ are self-conjugate and the following fusion rules hold.

$$
\begin{aligned}
{\left[\rho_{2 n}\right]\left[\rho_{2 n}\right] } & =[i d] \oplus\left[\rho_{4}\right] \oplus \cdots \oplus\left[\rho_{2 n-4}\right] \oplus\left[\rho_{2 n}\right], \\
{\left[\rho_{2 n}\right]\left[\rho_{2 n+1}\right] } & =\left[\rho_{2}\right] \oplus\left[\rho_{6}\right] \oplus \cdots \oplus\left[\rho_{2 n-2}\right] .
\end{aligned}
$$

Proof. Suppose that $n$ is odd and $\left[\rho_{2 n}\right]$ is self-conjugate. As in the proof of Theorem 3.7, the following fusion rules hold.

$$
\begin{aligned}
{\left[\rho_{2 n}\right]\left[\rho_{2 n}\right] } & =[i d] \oplus\left[\rho_{4}\right] \oplus \cdots \oplus\left[\rho_{2 n-6}\right] \oplus\left[\rho_{2 n-2}\right], \\
{\left[\rho_{2 n}\right]\left[\rho_{2 n+1}\right] } & =\left[\rho_{2}\right] \oplus\left[\rho_{6}\right] \oplus \cdots \oplus\left[\rho_{2 n-4}\right] \oplus\left[\rho_{2 n+1}\right] .
\end{aligned}
$$

Since we assume that $\left[\rho_{2 n}\right]$ is self-conjugate, $\left[\rho_{2 n+1}\right]$ is also self-conjugate. By conjugating the second equality, we obtain

$$
\left[\rho_{2 n+1}\right]\left[\rho_{2 n}\right]=\left[\rho_{2}\right] \oplus\left[\rho_{6}\right] \oplus \cdots \oplus\left[\rho_{2 n-4}\right] \oplus\left[\rho_{2 n+1}\right] .
$$

On the other hand, from the symmetry between $\left[\rho_{2 n}\right]$ and $\left[\rho_{2 n+1}\right]$, we also obtain

$$
\left[\rho_{2 n}\right]\left[\rho_{2 n+1}\right]=\left[\rho_{2}\right] \oplus\left[\rho_{6}\right] \oplus \cdots \oplus\left[\rho_{2 n-4}\right] \oplus\left[\rho_{2 n}\right] \text {. }
$$

But this is impossible because $\left[\rho_{2 n}\right]$ and $\left[\rho_{2 n+1}\right]$ are different sectors. (If $\left[\rho_{2 n}\right]=$ $\left[\rho_{2 n+1}\right]$, the principal graph is not $D_{2 n+2}$.)

Next, suppose that $n$ is even and $\left[\rho_{2 n+1}\right]=\left[\bar{\rho}_{2 n}\right]$. In the same way, we have 


$$
\begin{aligned}
{\left[\rho_{2 n}\right]\left[\rho_{2 n}\right] } & =\left[\rho_{2}\right] \oplus\left[\rho_{6}\right] \oplus \cdots \oplus\left[\rho_{2 n-2}\right], \\
{\left[\rho_{2 n}\right]\left[\rho_{2 n+1}\right] } & =[i d] \oplus\left[\rho_{4}\right] \oplus \cdots \oplus\left[\rho_{2 n-4}\right] \oplus\left[\rho_{2 n}\right] .
\end{aligned}
$$

By conjugating the second equality, we obtain

$$
\left[\rho_{2 n}\right]\left[\rho_{2 n+1}\right]=[i d] \oplus\left[\rho_{4}\right] \oplus \cdots \oplus\left[\rho_{2 n-4}\right] \oplus\left[\rho_{2 n+1}\right] .
$$

And we get a cotradiction. By the same argument as in the proof of Theorem 3.7, we have the fusion rules.

Q.E.D.

The difference between $D_{4 n}$ and $D_{4 n+2}$ we saw above is stated by $\mathrm{A}$. Ocneanu in [01, page 157], but no proof has been published until now.

We compute the statistical dimensions of the right hand sides of fusion rules in Proposition 3.10.

The case of odd $n$ :

$$
\begin{aligned}
\sum_{k=1}^{(n-1) / 2} d_{\rho_{4 k-2}}+d_{\rho_{2 n+1}} & =\sum_{k=1}^{(n-1) / 2} \frac{\sin \frac{4 k-1}{4 n+2} \pi}{\sin \frac{\pi}{4 n+2}}+\frac{1}{2 \sin \frac{\pi}{4 n+2}} \\
& =\frac{1}{\sin \frac{\pi}{4 n+2}\left[\sum_{k=1}^{(n+1) / 2} \sin \frac{4 k-1}{4 n+2} \pi-\frac{1}{2}\right],} \\
\sum_{k=1}^{(n+1) / 2} d_{\rho_{4 k-4}} & =\sum_{k=1}^{(n+1) / 2} \frac{\sin \frac{4 k-3}{4 n+2} \pi}{\sin \frac{\pi}{4 n+2}} .
\end{aligned}
$$

It is easy to show

$$
\sum_{k=1}^{(n+1) / 2}\left(\sin \frac{4 k-1}{4 n+2} \pi-\sin \frac{4 k-3}{4 n+2} \pi\right)=\frac{1}{2} .
$$

The case of even $n$ :

$$
\begin{aligned}
\sum_{k=1}^{n / 2} d_{\rho_{4 k-4}}+d_{\rho_{2 n+1}} & =\frac{1}{\sin \frac{\pi}{4 n+2}}\left[\sum_{k=1}^{n / 2} \sin \frac{4 k-3}{4 n+2} \pi+\frac{1}{2}\right] \\
\sum_{k=1}^{n / 2} d_{\rho_{4 k-2}} & =\frac{1}{\sin \frac{\pi}{4 n+2}}\left[\sum_{k=1}^{n / 2} \sin \frac{4 k-1}{4 n+2} \pi\right]
\end{aligned}
$$

It is also easy to show 


$$
\sum_{k=1}^{n / 2}\left(\sin \frac{4 k-1}{4 n+2} \pi-\sin \frac{4 k-3}{4 n+2} \pi\right)=\frac{1}{2}
$$

Then there is no contradiction.

The principal graph of $M \supset \rho_{2}(M)$ is as in Fig. 15 .

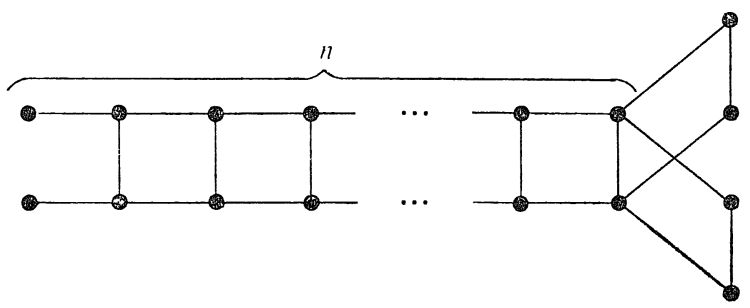

Fig. 15. The principal graph of $M \supset \rho_{2}(M)$ in the case of $D_{2 n+2} n \geq 2$.

\section{§4. Characterization of Fixed Point Algebra and Subfactors with Depth 2}

First we prove a theorem of H. Kosaki, A. Ocneanu, M. Pimsner and S. Popa [K3] [PP1, Corollary 1.1.6] by using sectors.

Theorem 4.1. Let $M \supset N$ be a pair of properly infinite factors with $[M: N]$ $<\infty$ and $M \cap N^{\prime}=C$. The following conditions are equivalent.

(i) There exists a finite group $G$ and its outer action $\alpha$ on $M$ such that $N=M^{\alpha}$ and $|G|=[M: N]_{0}$.

(ii) If $r: M \rightarrow N$ is the canonical endomorphism and $[\gamma]=\bigoplus_{i}\left[\rho_{i}\right]$ is irreducible decomposition, then $d_{\rho_{i}}=1$, i.e. $[r]$ is decomposed into automorphisms.

If the above conditions are satisfied, then

$$
[r]=\bigoplus_{g \in G}\left[\alpha_{g}\right] .
$$

Proof. (i) $\Rightarrow$ (ii): We assume (i) and define $E \in \mathrm{E}(M, N)$ by

$$
E(x)=\frac{1}{|G|} \sum_{g \in G} \alpha_{g}(x) \text { for } x \in M .
$$

In Subsection 2.3, we saw that from $E$ we can construct an $M-M$ correspondence and, by using the bijection between $\operatorname{Sect}(\mathrm{M})$ and the set of unitary equivalence classes of $M-M$ correspondences, we obtain $\rho_{E} \in \operatorname{End}(M)$ such that $\left[\rho_{E}\right]=[r]$. We show $\left[\rho_{E}\right]=\bigoplus_{g \in G}\left[\alpha_{g}\right]$. Let $\Omega$ be a cyclic and separating vector for $M$ (and equivalently $M^{\prime}$ ) and $\left\{S_{g}\right\}_{g \in G} \subset M$ be isometries satisfying $\sum_{g \in G} S_{g} S_{g}^{*}=1$. We define $\rho^{\prime} \in \operatorname{End}(M)$ by 


$$
\rho^{\prime}(x) \equiv \sum_{g \in G} S_{g} \alpha_{g}(x) S_{g}^{*}
$$

Then

$$
\begin{gathered}
\langle E(x) \Omega y, \Omega\rangle=\frac{1}{|G|} \sum_{g \in G}\left\langle\alpha_{g}(x) \Omega y, \Omega\right\rangle=\left\langle\rho^{\prime}(x) \xi y, \xi\right\rangle, \\
\text { where } \xi \equiv \frac{1}{\sqrt{|G|}} \sum_{g \in G} S_{g} \Omega
\end{gathered}
$$

It suffices to show $\overline{\rho^{\prime}(M) \xi M}=H$. Since $\alpha$ is an outer action, $M \cap \rho^{\prime}(M)^{\prime}=$ $\bigoplus_{g \in G} C S_{g} S_{g}^{*}$, and the projection $p$ onto $\overline{\rho^{\prime}(M) \xi M}$ belongs to $\bigoplus_{g \in G} \mathbb{C} S_{g} S_{g}^{*}$. From $S_{g} S_{g}^{*} \xi=\frac{1}{\sqrt{|G|}} S_{g} \Omega \neq 0, p$ can not be orthogonal to $S_{g} S_{g}^{*}$ for any $g \in G$, which implies $p=1$. Hence we obtain $\overline{\rho^{\prime}(M) \xi M}=H$ and so we have $\left[\rho_{E}\right]=$ $\bigoplus_{g \in G}\left[\alpha_{g}\right]$.

(ii) $\Rightarrow$ (i): First we assume (ii) and $M \simeq N$. We take $\rho, \bar{\rho} \in \operatorname{End}(M)$ such that $\rho(M)=N$. From Subsection 2.2,

$$
[r]=[\rho \bar{\rho}]=\bigoplus_{i}\left[\rho_{i}\right] .
$$

Since $[\rho \bar{\rho}]$ is self-conjugate, $\left\{\left[\rho_{i}\right]\right\}$ is a self-conjugate set, i.e. $\left\{\left[\rho_{i}\right]\right\}$ is invariant under inverse because conjugation and inverse are equivalent on $\operatorname{Out}(M)$. Multiplying both sides of (4.1.1) by $\left[\rho_{j}\right]$ from left, we get

$$
\left[\rho_{j} \rho\right][\bar{\rho}]=\bigoplus_{i}\left[\rho_{j}\right]\left[\rho_{i}\right]
$$

and, from above observation, it follows that the right hand side of this equality contains $[i d]$. Since $\left[\rho_{j} \rho\right]$ is irreducible because $[\rho]$ is irreducible and $\left[\rho_{j}\right]$ is an automorphism, this means that $\left[\rho_{j} \rho\right]=[\rho]$ by the characterization of the conjugate sector in Subsection 2.4 and hence

$$
\bigoplus_{i}\left[\rho_{j}\right]\left[\rho_{i}\right]=\bigoplus_{i}\left[\rho_{i}\right] .
$$

Since $[\rho \bar{\rho}]$ contains $[i d]$ with multiplicity one, every $\left[\rho_{i}\right]$ is different and $\left\{\left[\rho_{i}\right]\right\}_{i}$ is a subgroup of $\operatorname{Out}(M)$. We write $G=\left\{\left[\rho_{i}\right]\right\}=\left\{\left[\alpha_{g}\right]\right\}_{g \in G}$.

Once we fix representative $\rho \in \operatorname{End}(M)$, we can take $\alpha_{g} \in \operatorname{Aut}(M)$ such that $\alpha_{g} \circ \rho=\rho$. This choice makes $\left\{\alpha_{g}\right\}$ an outer action of $G$. Indeed, $\alpha_{g} \circ \alpha_{g^{\prime}} \circ \alpha_{g g^{\prime}}^{-1}$ is inner for $g, g^{\prime} \in G$, and

$$
\alpha_{g} \circ \alpha_{g^{\prime}} \circ \alpha_{g^{\prime}}^{-1} \circ \rho=\alpha_{g} \circ \alpha_{g^{\prime}} \circ \rho=\alpha_{g} \circ \rho=\rho .
$$

Since $\rho$ is irreducible, this means $\alpha_{g^{\prime}} \circ \alpha_{g^{\prime}} \circ \alpha_{g g^{\prime}}^{-1}=i d$. So $\left\{\alpha_{g}\right\}$ is an action of $G$ and $M^{G} \supset \rho(M)$. By comparing indices, we get $\rho(M)=M^{G}$ and have (i). 
In a general case (where $M \simeq N$ does not necessarily hold), we define $\hat{\rho} \in$ $\operatorname{End}(M \otimes N)$ by

$$
\hat{\rho}(x \otimes y) \equiv y \otimes r(x) \quad \text { for } \quad x \in M, y \in N .
$$

It is easy to see that $\hat{\rho}$ is irreducible. $\hat{\rho}$ is self-conjugate because

$$
\left.\hat{r} \equiv r \otimes r\right|_{N}: M \otimes N \rightarrow N \otimes r(M)
$$

is the canonical endomorphism and the equality $\hat{\gamma}=\hat{\rho}^{2}$ holds. By the characterization of conjugate sector in Subsection 2.4, $[\rho]^{2}=[r] \otimes\left[\left.r\right|_{N}\right]=\oplus_{i}\left[\rho_{i}\right] \otimes\left[\left.r\right|_{N}\right]$ must contain $\left[i d_{M}\right] \otimes\left[i d_{N}\right]$ with multiplicity one. Therefore one of $\left[\rho_{i}\right]$ is $\left[i d_{M}\right]$ and, $\left[\left.r\right|_{N}\right]$ contains $\left[i d_{N}\right]$ with multiplicity one. By the same argument as in the case of $M \simeq N$, we see that $G \equiv\left\{\left[\rho_{i}\right] \otimes\left[i d_{N}\right]\right\}$ is a group and we get an outer action $\left\{\alpha_{g}\right\} \subset \operatorname{Aut}(M)$ such that $\left\{\left[\alpha_{g}\right]\right\}=\left\{\left[\rho_{i}\right]\right\}$ and $\left(\alpha_{g} \otimes i d_{N}\right) \circ \hat{\rho}=\hat{\rho}$. Due to (4.1.2), we obtain $N \subset M^{G}$. By comparing indices, we get $N=M^{G}$. Q.E.D.

In a sense, the following proposition is a generalization of Thoerem 4.1.

Proposition 4.2. Let $M$ be a properly infinite factor and $\rho \in \operatorname{End}(M)$ with $d_{\rho}<\infty$ and $M \cap \rho(M)^{\prime}=C$. If $[\rho \bar{\rho}]=\bigoplus_{i} n_{i}\left[\alpha_{i}\right]$ and $[\bar{\rho} \rho]=\bigoplus_{a} m_{a}\left[\beta_{a}\right]$ are irreducible decompositions where $n_{i}$ and $m_{a}$ are the multiplicities, then the following conditions are equivalent.

(i) The depth of $M \supset \rho(M)$ is two.

(ii) The depth of $M \supset \bar{\rho}(M)$ is two.

(iii) $M \cap \rho \bar{\rho} \rho(M)^{\prime}$ is a factor.

(iv) $M \cap \bar{\rho} \rho \bar{\rho}(M)^{\prime}$ is a factor.

(v) $n_{i}=d_{\alpha_{i}}$ for all $i$.

(vi) $m_{a}=d_{\beta_{a}}$ for all $a$.

(vii) $\left[\alpha_{i}\right][\rho]=d_{\alpha_{i}}[\rho]$ for all $i$.

(viii) $\left[\beta_{a}\right][\bar{\rho}]=d_{\beta_{a}}[\bar{\rho}]$ for all $a$.

Proof. (i) $\Leftrightarrow$ (iii) and (ii) $\Leftrightarrow$ (iv) are obvious from the definition of depth 2 and (iii) $\Leftrightarrow$ (iv) follows from $[\overline{\rho \bar{\rho} \rho}]=[\bar{\rho} \rho \bar{\rho}] . \quad$ It is enough to show (iii) $\Leftrightarrow($ vii) $\Leftrightarrow(\mathrm{v})$ because (iv) $\Leftrightarrow$ (viii) $\Leftrightarrow$ (vi) is shown in the same way.

(iii) $\Rightarrow$ (vii): (iii) means that $[\rho \bar{\rho} \rho]$ is a multiple of an irreducible sector. Since $[\rho \bar{\rho}]$ contains $[i d],[\rho \bar{\rho} \rho]$ must be a multiple of $[\rho]$. Then

$$
[\rho \bar{\rho} \rho]=\bigoplus_{i} n_{i}\left[\alpha_{i}\right][\rho]=d_{\rho}^{2}[\rho]
$$

and hence $\left[\alpha_{i}\right][\rho]=d_{\alpha_{i}}[\rho]$, where the multiplicity $d_{\alpha_{i}}$ has been determined by 
the relation $d_{\alpha_{i} \circ \rho}=d_{\alpha_{i}} d_{\rho}$ due to the multiplicativity of the statistical dimension. (vii) $\Rightarrow(\mathrm{v}):$ Multiplying $[\rho \bar{\rho}]=\bigoplus_{i} n_{i}\left[\alpha_{i}\right]$ by $\left[\bar{\alpha}_{i}\right]$ from left, we have

$$
d_{\alpha_{j}}[\rho][\bar{\rho}]=\left[\bar{\alpha}_{j}\right][\rho][\bar{\rho}]=\bigoplus_{i} n_{i}\left[\bar{\alpha}_{j}\right]\left[\alpha_{i}\right]
$$

because $\left[\bar{\alpha}_{j}\right]$ must be an element $\left[\alpha_{i}\right]$ of the self-conjugate set $\left\{\left[\alpha_{i}\right]\right\}$ and $d_{\alpha_{j}}=d_{\bar{\alpha}_{j}}$. This means that the right hand side contains [id] with multiplicity $d_{\alpha^{\prime} j}$ and so $n_{j}=d_{\alpha_{j}}$.

(v) $\Rightarrow$ (iii): Since $\left[\bar{\alpha}_{j}\right][\rho \bar{\rho}]=\bigoplus_{i} d_{\alpha_{i}}\left[\bar{\alpha}_{j}\right]\left[\alpha_{i}\right]$ contains [id] with multiplicity $d_{\alpha_{j} j}$, $\left[\bar{\alpha}_{j}\right][\rho]$ must contain $[\rho]$ with the same multiplicity. But we have $d_{\bar{\alpha}_{j \circ \rho}}=d_{\bar{\alpha}_{j}} d_{\rho}$ from the multiplicativity of the statistical dimension. Therefore, $\left[\bar{\alpha}_{j}\right][\rho]=$ $d_{\alpha j}[\rho]$

Q.E.D.

The relation $\left[\rho_{i}\right][\rho]=[\rho]$ means that $\rho(M)$ is included in $M^{\rho i}$ for some representative $\rho_{i}$ of $\left[\rho_{i}\right]$. So if $\rho$ satisfies the conditions of Proposition 4.2, in particular (vii), $\rho(M)$ is a kind of generalized fixed point algebra. In fact, A. Ocneanu announced that depth of $M \supset N$ is 2 if and only if the relation of $M \supset N$ is the crossed product by a Kac algebra action.

For more detailed analysis, we need intertwiners. Let $\rho_{1}, \rho_{2} \in \operatorname{End}(M)$. We define $\mathrm{H}\left(\rho_{1}, \rho_{2}\right)$ as the set of intertwiners from $\rho_{1}$ to $\rho_{2}$ :

$$
\mathbb{H}\left(\rho_{1}, \rho_{2}\right) \equiv\left\{v \in M ; v \rho_{1}(x)=\rho_{2}(x) v \text { for } x \in M\right\} \text {. }
$$

If $\rho_{1}$ is irreducible, $\mathrm{H}\left(\rho_{1}, \rho_{2}\right)$ is a Hilbert space with $\left(v_{1}, v_{2}\right) \equiv v_{2}^{*} v_{1}, v_{1}, v_{2} \in$ $\mathrm{H}\left(\rho_{1}, \rho_{2}\right)$. Indeed, since $v_{2}^{*} \in \mathrm{H}\left(\rho_{2}, \rho_{1}\right)$,

$$
v_{2}^{*} v_{1} \rho_{1}(x)=v_{2}^{*} \rho_{2}(x) v_{1}=\rho_{1}(x) v_{2}^{*} v_{1}
$$

and so $v_{2}^{*} v_{1} \in \mathbb{C}$. We write $\mathrm{H}(\rho) \equiv \mathrm{H}(i d, \rho)$.

When $[\rho \bar{\rho}]=\bigoplus_{g \in G}\left[\alpha_{g}\right]$ i.e. " $[\rho \bar{\rho}]$ is decomposed into elements of $G$ ", it is natural to ask what $[\bar{\rho} \rho]$ is. The answer: " $[\bar{\rho} \rho]$ is decomposed into elements of $\hat{G}$ ". We show this more precisely. Since we assume the conditions of Theorem 4.1, $\rho$ satisfies the conditions of Proposition 4.2. So the irreducible decomposition of $[\bar{\rho} \rho]$ is as follows:

$$
[\bar{\rho} \rho]=\oplus d_{\sigma}\left[\beta_{\sigma}\right] \quad \text { where } \quad d_{\sigma} \equiv d_{\beta_{\sigma}}
$$

and $\left[\beta_{\sigma}\right][\bar{\rho}]=d_{\sigma}[\bar{\rho}]$ is satisfied. By conjugating $\left[\alpha_{g}\right][\rho]=[\rho]$, we have $[\bar{\rho}]\left[\alpha_{g}\right]=$ $[\bar{\rho}]$ and this means that there is a unitary $\lambda_{g} \in M$ such that $\lambda_{g} \in \mathbb{H}\left(\bar{\rho}, \bar{\rho} \circ \alpha_{g}\right)$. In fact, a stronger statement holds. From the duality of the fixed point algebra and the crossed product [J, page 7], 


$$
M_{1}=M \rtimes_{\alpha} G
$$

where $M_{1}$ is the extension of $M$ by $\rho(M)$. On the other hand, there exists $\hat{\rho} \in \operatorname{End}\left(M_{1}\right)$, an extension of $\bar{\rho}$, which satisfies $\hat{\rho}\left(M_{1}\right)=M[\mathrm{~L} 2$, page 296], and so

$$
M=\bar{\rho}(M) \underset{\bar{\rho} \circ \alpha_{\circ} \bar{\rho}^{-1}}{\rtimes} G
$$

Then there exists a unitary representation $\left\{\lambda_{g}\right\}_{g \in G} \subset M$ such that

$$
\bar{\rho} \circ \alpha_{g}(x)=\left(\bar{\rho} \circ \alpha_{g} \circ \bar{\rho}^{-1}\right)(\bar{\rho}(x))=\operatorname{Ad} \lambda_{g}(\bar{\rho}(x))
$$

and $\left(\bar{\rho}(M) \cup\left\{\lambda_{g}\right\}_{g \in G}\right)^{\prime \prime}=M$.

Let $\left\{W(\sigma)_{a}\right\}$ be an orthonormal basis of $\mathrm{H}\left(\bar{\rho}, \beta_{\sigma} \circ \bar{\rho}\right)$ and we define matrix elements $\beta_{\sigma}(x)_{a b} \equiv W(\sigma)_{a}^{*} \beta_{\sigma}(x) W(\sigma)_{b}$. By the definition of $\mathrm{H}\left(\bar{\rho}, \beta_{\sigma} \circ \bar{\rho}\right)$, $\beta_{\sigma}(\bar{\rho}(x))_{a b}=\delta_{a b} \bar{\rho}(x)$, i.e. $\bar{\rho}(M)$ is diagonal. Since

$$
\begin{aligned}
\beta_{\sigma}\left(\lambda_{g}\right)_{a b} \bar{\rho}(x) & =\sum_{c} \beta_{\sigma}\left(\lambda_{g}\right)_{a c} \delta_{c b} \bar{\rho}(x)=\beta_{\sigma}\left(\lambda_{g} \bar{\rho}(x)\right)_{a b}=\beta_{\sigma}\left(\bar{\rho} \circ \alpha_{g}(x) \lambda_{g}\right)_{a b} \\
& =\sum_{c} \delta_{a c} \bar{\rho} \circ \alpha_{g}(x) \beta_{\sigma}\left(\lambda_{g}\right)_{c b}=\bar{\rho} \circ \alpha_{g}(x) \beta_{\sigma}\left(\lambda_{g}\right)_{a b},
\end{aligned}
$$

$\beta_{\sigma}\left(\lambda_{g}\right)_{a b}$ is proportional to $\lambda_{g}$ and we write

$$
\beta_{\sigma}\left(\lambda_{g}\right)_{a b}=\pi\langle g, \sigma\rangle_{a b} \lambda_{g} .
$$

It is easy to see that the matrices $\pi\langle\cdot, \sigma\rangle$ form a unitary representation of G. Moreover, it is irreducible for each $\sigma$ and if $\sigma_{1} \neq \sigma_{2}$, then $\pi\left\langle\cdot, \sigma_{1}\right\rangle$ and $\pi\left\langle\cdot, \sigma_{2}\right\rangle$ are disjoint. Indeed, if $\pi\langle\cdot, \sigma\rangle$ were reducible, by changing the basis of $\mathrm{H}\left(\bar{\rho}, \beta_{\sigma} \circ \bar{\rho}\right)$, we would have

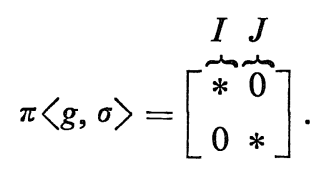

By easy computation, we can check

$$
\sum_{a \in I} W(\sigma)_{a} W(\sigma)_{a}^{*} \in\left\langle\beta_{\sigma}\left(\lambda_{G}\right), \beta_{\sigma}(\bar{\rho}(M))\right\rangle^{\prime} \cap M=\beta_{\sigma}(M)^{\prime} \cap M .
$$

Since $\beta_{\sigma}$ is irreducible, this implies $\sum_{a \in I} W(\sigma)_{a} W(\sigma)_{a}^{*}=1$ and $J$ must be empty. If $\pi\left\langle\cdot, \sigma_{1}\right\rangle$ and $\pi\left\langle\cdot, \sigma_{2}\right\rangle$ are equivalent, we may assume that $\pi\left\langle\cdot, \sigma_{1}\right\rangle=\pi\left\langle\cdot, \sigma_{2}\right\rangle$ by a change of basis. We define $U=\sum_{a} W\left(\sigma_{1}\right)_{a} W\left(\sigma_{2}\right)_{a}^{*}$. Then $U$ is unitary and $U \beta_{\sigma_{2}}(x)=\beta_{\sigma_{1}}(x) U$, i.e. $\left[\beta_{\sigma_{1}}\right]=\left[\beta_{\sigma_{2}}\right]$.

Note that $\operatorname{dim} \pi\langle\cdot, \sigma\rangle=d_{\sigma}$ is the multiplicity of $\left[\beta_{\sigma}\right]$ in $[\bar{\rho} \rho]$ and $\sum_{\sigma} d_{\sigma}^{2}=|G|$. This means that $\bigoplus_{\sigma} d_{\sigma} \cdot \pi\langle\cdot, \sigma\rangle$ is equivalent to the regular representation of $G$. 
In fact we see the coaction of $G_{0}$ We write $[\bar{\rho} \rho]=\bigoplus_{\sigma \in \hat{G}} d_{\sigma}\left[\beta_{\sigma}\right]$.

Proposition 4.3. Let $M$ be a properly infinite factor and $\rho \in \operatorname{End}(M)$ with $[\rho \bar{\rho}]=\bigoplus_{g \in G}\left[\alpha_{g}\right]$ where $\alpha$ is an outer action of a finite group $G$. Let $\beta_{\sigma}, d_{\sigma}$ and $\pi\langle g, \sigma\rangle$ be as above. Then,

(i) Each $\pi\langle\circ, \sigma\rangle$ is an irreducible unitary representation.

(ii) If $\sigma_{1} \neq \sigma_{2}$, then $\pi\left\langle\cdot, \sigma_{1}\right\rangle$ and $\pi\left\langle\circ, \sigma_{2}\right\rangle$ are disjoint.

(iii) If $\sigma_{1} \otimes \sigma_{2} \simeq \bigoplus_{i} n_{i} \circ \sigma_{i}$ is the irreducible decomposition where $n_{i}$ is the multiplicity of $\sigma_{i}$, then $\left[\beta_{\sigma_{1}}\right]\left[\beta_{\sigma_{2}}\right]=\bigoplus_{i} n_{i} \circ\left[\beta_{i}\right]$.

Proof. It suffices to show (iii). Since depth of $M \supset \bar{\rho}(M)$ is 2 , the irreducible components of $\left[\beta_{\sigma_{1}}\right]\left[\beta_{\sigma_{2}}\right]$ belong to $\left\{\left[\beta_{\sigma}\right]\right\}_{\sigma \in \hat{G}}$. We start from $\left[\beta_{\sigma_{1}}\right]\left[\beta_{\sigma_{2}}\right]$ $=\bigoplus_{i} n_{i}\left[\beta_{\sigma_{i}}\right]$ and prove $\sigma_{1} \otimes \sigma_{2} \simeq \bigoplus_{i} n_{i} \circ \sigma_{i}$. For simplicity we sometimes omit " $\sigma$ ". Since $\left[\beta_{1}\right]\left[\beta_{2}\right][\bar{\rho}]=d_{1} d_{2}[\bar{\rho}], \operatorname{dim} \mathbb{H}\left(\bar{\rho}, \beta_{1} \circ \beta_{2} \circ \bar{\rho}\right)=d_{1} d_{2}$. We have two different basis of $\mathbb{H}\left(\bar{\rho}, \beta_{1} \circ \beta_{2} \circ \bar{\rho}\right)$. Let $\left\{U\left(\sigma_{i}\right)_{s}\right\}_{s}$ be a basis of $\mathbb{H}\left(\beta_{i}, \beta_{1} \circ \beta_{2}\right)$. Then $\left\{U\left(\sigma_{i}\right)_{s} W\left(\sigma_{i}\right)_{e}\right\}_{i, s, e}$ is a basis of $\mathrm{H}\left(\bar{\rho}, \beta_{1} \circ \beta_{2} \circ \bar{\rho}\right)$. On the other hand it is easy to check that $\left\{\beta_{1}\left(W\left(\sigma_{2}\right)_{b}\right) W\left(\sigma_{1}\right)_{a}\right\}_{a, b}$ is another bais of $\mathrm{H}\left(\bar{\rho}, \beta_{1} \circ \beta_{2} \circ \bar{\rho}\right)$. Since

$$
\begin{aligned}
& W\left(\sigma_{1}\right)_{a}^{*} \beta_{1}\left(W\left(\sigma_{2}\right)_{b}^{*}\right) \beta_{1} \circ \beta_{2}\left(\lambda_{g}\right) \beta_{1}\left(W\left(\sigma_{2}\right)_{d}\right) W\left(\sigma_{1}\right)_{c} \\
& \quad=W\left(\sigma_{1}\right)_{a}^{*} \beta_{1}\left(W\left(\sigma_{2}\right)_{b}^{*} \beta_{2}\left(\lambda_{g}\right) W\left(\sigma_{2}\right)_{d}\right) W\left(\sigma_{1}\right)_{c} \\
& \quad=W\left(\sigma_{1}\right)_{a}^{*} \beta_{1}\left(\pi\left\langle g, \sigma_{2}\right\rangle_{b d} \lambda_{g}\right) W\left(\sigma_{1}\right)_{c} \\
& \quad=\pi\left\langle g, \sigma_{1}\right\rangle_{a c} \pi\left\langle g, \sigma_{2}\right\rangle_{b d} \lambda_{g}
\end{aligned}
$$

and

$$
\begin{aligned}
& W\left(\sigma_{i}\right)_{e}^{*} U\left(\sigma_{i}\right)_{s}^{*} \beta_{1} \circ \beta_{2}\left(\lambda_{g}\right) U\left(\sigma_{j}\right)_{t} W\left(\sigma_{j}\right)_{f} \\
& \quad=\delta_{i j} \delta_{s t} W\left(\sigma_{i}\right)_{e}^{*} \beta_{i}\left(\lambda_{g}\right) W\left(\sigma_{j}\right)_{f} \\
& =\delta_{i j} \delta_{s t} \pi\left\langle g, \sigma_{i}\right\rangle_{e f} \lambda_{g}
\end{aligned}
$$

we obtain $\sigma_{1} \otimes \sigma_{2} \simeq \bigoplus_{i} n_{i} \circ \sigma_{i}$.

Q.E.D.

Corollary 4.4. Let $M$ be a properly infinite factor and $N$ be the fixed point algebra of $M$ under an outer action of a non-commutative finite group $G$. Then there exists no self-conjugate endomorphism $\rho$ such that $\rho(M)=N$.

Proof. From Proposition 4.3, we get $[\rho \bar{\rho}] \neq[\bar{\rho} \rho]$ because $d_{\sigma}>1$ for some $\sigma$ if $G$ is non-commutative and hence $[\rho] \neq[\bar{\rho}]$.

Q.E.D.

For later use, we generalize Proposition 4.3. Let $M$ be a properly infinite factor and $\rho \in \operatorname{End}(M)$. Assume that $\rho$ satisfies the conditions of Propositions 4.2, i.e. the depth of $M \supset \rho(M)$ is two. Then the irreducible decomposi- 
tions of $[\rho \bar{\rho}]$ and $[\bar{\rho} \rho]$ are

$$
[\rho \bar{\rho}]=\otimes d_{g}\left[\alpha_{g}\right], \quad[\bar{\rho} \rho]=\oplus d_{\sigma}\left[\beta_{\sigma}\right] .
$$

Let $\left\{V(g)_{i}\right\}_{i}$ and $\left\{W(\sigma)_{a}\right\}_{a}$ be basis of $\mathrm{H}\left(\bar{\rho}, \bar{\rho} \circ \alpha_{g}\right)$ and $\mathrm{H}\left(\bar{\rho}, \beta_{\sigma} \circ \bar{\rho}\right)$, and $\beta_{\sigma}(x)_{a b} \equiv$ $W(\sigma)_{a}^{*} \beta_{\sigma}(x) W(\sigma)_{b}$ as before. Then $\beta_{\sigma}\left(V(g)_{i}\right)_{a b} \bar{\rho}(x)=\bar{\rho} \circ \alpha_{g}(x) \beta_{\sigma}\left(V(g)_{i}\right)_{a b}$ and hence $\beta_{\sigma}\left(V(g)_{i}\right)_{a b}$ is a linear combination of $\left\{V(g)_{i}\right\}_{i}$. We write $\beta_{\sigma}\left(V(g)_{i}\right)_{a b}=$ $\sum_{j} \pi_{a b}^{j i}\langle g, \sigma\rangle V(g)_{j}$. We use the following convention.

$$
\begin{aligned}
\left(\pi_{a b}^{\cdot}\langle g, \sigma\rangle\right)_{i j} & =\pi_{a b}^{i j}\langle g, \sigma\rangle \in M\left(d_{g}\right) . \\
\left(\pi_{\bullet}^{i j}\langle g, \sigma\rangle\right)_{a b} & =\pi_{a b}^{i j}\langle g, \sigma\rangle \in M\left(d_{\sigma}\right) . \\
\left(\pi_{:}^{*}\langle g, \sigma\rangle\right)_{i a, j b} & =\pi_{a b}^{i j}\langle g, \sigma\rangle \in M\left(d_{g} d_{\sigma}\right) . \\
\left(\pi_{\bullet}^{t r}\langle g, \sigma\rangle\right)_{a b} & =\sum_{i} \pi_{a b}^{i i}\langle g, \sigma\rangle \in M\left(d_{\sigma}\right) . \\
\left(\pi_{i r}^{*}\langle g, \sigma\rangle\right)_{i j} & =\sum_{a} \pi_{a a}^{i j}\langle g, \sigma\rangle \in M\left(d_{g}\right) .
\end{aligned}
$$

Note that $\left[\alpha_{g_{1}}\right]\left[\alpha_{g_{2}}\right]$ and $\left[\beta_{\sigma_{1}}\right]\left[\beta_{\sigma_{2}}\right]$ are always decomposed into $\left\{\left[\alpha_{g}\right]\right\}$ and $\left\{\left[\beta_{\sigma}\right]\right\}$.

Proposition 4.5. Let $M$ be a properly infinite factor and $\rho \in \operatorname{End}(M)$ such that the depth of $M \supset \rho(M)$ is two. If $\left\{V(g)_{i}\right\}_{i},\left\{W(\sigma)_{a}\right\}_{a}$ and $\pi_{a b}^{i j}\langle g, \sigma\rangle$ are as above, the following statements hold.

(i) $\pi_{0}:\langle g, \sigma\rangle$ is unitary.

(ii) If $\left[\alpha_{g_{1}}\right]\left[\alpha_{g_{2}}\right]=\bigoplus_{i} n_{i}\left[\alpha_{g_{i}}\right]$ then $\sum_{c} \pi_{a c}^{\cdot}\left\langle g_{1}, \sigma\right\rangle \otimes \pi_{c b}^{\cdot}\left\langle g_{2}, \sigma\right\rangle$ is unitarily equivalent to $\bigoplus_{i} \pi_{a b}^{\cdot}\left\langle g_{i}, \sigma\right\rangle \otimes 1_{n_{i} \cdot}$. Moreover, there is a unitary which induces the above equivalence simultaneously for every pair $(a, b)$.

(iii) If $\left[\beta_{\sigma_{1}}\right]\left[\beta_{\sigma_{2}}\right]=\bigoplus_{i} m_{i}\left[\beta_{\sigma_{i}}\right]$, then $\sum_{l} \pi_{\bullet}^{j l}\left\langle g, \sigma_{1}\right\rangle \otimes \pi_{\bullet}^{l k}\left\langle g, \sigma_{2}\right\rangle$ is unitarily equivalent to $\bigoplus_{i} \pi_{\cdot}^{j k}\left\langle g, \sigma_{i}\right\rangle \otimes 1_{m_{i}}$. Moreover, there is a unitary which induces the above equivalence simultaneously for every pair $(j, k)$.

(iv) For fixed $\sigma, g \rightarrow \pi_{\cdot}^{t r}\langle g, \sigma\rangle$ is a representation of the fusion rules of $\left\{\left[\alpha_{g}\right]\right\}$, i.e. $\left[\alpha_{g}\right] \rightarrow \pi^{t r}\langle g, \sigma\rangle$ preserves the sum and the product.

(v) For fixed $g, \sigma \rightarrow \pi_{i r}^{*}\langle g, \sigma\rangle$ is a representation of the fusion rules of $\left\{\left[\beta_{\sigma}\right]\right\}$.

(vi) $M$ is generated by $\bar{\rho}(M)$ and $\left\{V(g)_{i}\right\}_{g, i}$.

Proof. (i): Since $\beta_{\sigma}\left(V(g)_{i}^{*}\right) \beta_{\sigma}\left(V(g)_{j}\right)=\delta_{i j}$

$$
\begin{aligned}
\delta_{i j} \delta_{a b} & =\sum_{c} \beta_{\sigma}\left(V(g)_{i}^{*}\right)_{a c} \beta_{\sigma}\left(V(g)_{j}\right)_{c b} \\
& =\sum_{c}\left(\sum_{k} \pi_{c a}^{k i}\langle g, \sigma\rangle V(g)_{k}\right)^{*}\left(\sum_{l} \pi_{c b}^{l j}\langle g, \sigma\rangle V(g)_{l}\right)
\end{aligned}
$$




$$
\begin{aligned}
& =\sum_{c, k, l} \overline{\pi_{c a}^{k i}\langle g, \sigma\rangle} \pi_{c b}^{l j}\langle g, \sigma\rangle V(g)_{k}^{*} V(g)_{l} \\
& =\sum_{c, k} \overline{\pi_{c a}^{k i}\langle g, \sigma\rangle} \sigma_{c b}^{k j}\langle g, \sigma\rangle .
\end{aligned}
$$

(ii) and (iii) can be proved in the same way as the proof of Proposition 4.3. (iv) and (v) are direct consequence of (ii) and (iii). (vi): Let $N$ be a von Neumann algebra generated by $\bar{\rho}(M)$ and $\left\{V(g)_{i}\right\}_{g, i}$. Let $v$ be an isometry in $\mathrm{H}(\bar{\rho} \rho)$ and $f \equiv v v^{*}$. Then $M$ is generated by $\bar{\rho}(M)$ and $f,(f$ is the conditional expectation from $\bar{\rho}(M)$ to $\bar{\rho} \rho(M)[\mathrm{L} 2]$ ), and it suffices to show that $v \in N$. Let $\left\{S(g)_{i}\right\}$ be a basis of $\mathrm{H}\left(\alpha_{g}, \rho \bar{\rho}\right)$. By comparing dimension, we see that $\left\{\bar{\rho}\left(S(g)_{i}\right) V(g)_{j}\right\}_{g, i, j}$ is a basis of $\mathrm{H}(\bar{\rho}, \bar{\rho} \rho \bar{\rho})$. Since $\mathrm{H}(\bar{\rho} \rho) \subset H(\bar{\rho}, \bar{\rho} \rho \bar{\rho})$, we get the desired result.

\section{§5. The Classification of the Subfactors of the Type $\mathbb{I I I}_{1} \mathrm{AFD}$}

\section{Factor with Index 3}

Let $R \supset P$ be a pair of type $\mathbb{I}_{1}$ AFD factors with $[R: P]=3$. The classification of such pairs is well-known [01, page 161], [P1]. If the principal graph is $D_{4}, P=R^{Z_{3}}$ and, if the principal graph is $A_{5}, R=Q \rtimes S_{3} \supset P=Q \rtimes S_{2}$ where $Q$ is a subfactor of $P$. The following theorem states that in the case of type III $_{1}$ AFD factors the situation is the same.

Theorem 5.1. Let $M$ be a type $I I I_{1}$ AFD factor and $N$ be a subfactor of $M$ with $[M: N]_{0}=3$. Then there exists a pair of type $I_{1} A F D$ factors $R \supset P$ such that $R \otimes M_{0} \supset P \otimes M_{0}$ is isomorphic to $M \supset N$ where $M_{0}$ is a type III $I_{1}$ AFD factor.

Proof. By a theorem of P. H. Loi [Li, Corollaire 2.4], $N$ is also a type III $_{1}$ AFD factor and there exists $\rho_{1} \in \operatorname{End}(M)$ such that $\rho_{1}(M)=N$. If the principal graph is $D_{4}$, by Theorem $4.1 N=M^{Z_{3}}$ and we get the stated result by the classification of group actions [KST, Theorem 20]. We now assume that the principal graph is $A_{5}$. Our aim is to look for a subfactor with index 6 and an $S_{3}$ action. The descendant sectors and fusion rules are as follows (see Fig. 16):

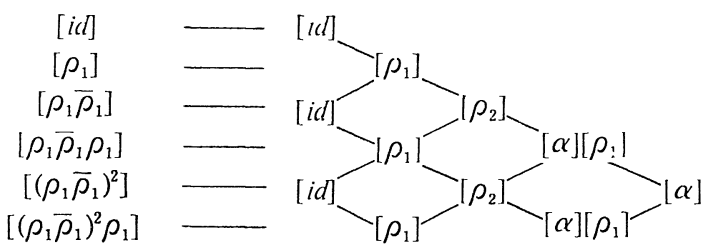

Fig. 16. The diagram of fusion rules in the case of $A_{5}$. 


$$
\begin{aligned}
{\left[\rho_{1}\right]\left[\bar{\rho}_{1}\right] } & =[i d] \oplus\left[\rho_{2}\right], \\
{\left[\rho_{2}\right]\left[\rho_{1}\right] } & =\left[\rho_{1}\right] \oplus[\alpha]\left[\rho_{1}\right], \\
{\left[\rho_{2}\right]^{2} } & =[i d] \oplus[\alpha] \oplus\left[\rho_{2}\right], \\
{[\alpha]\left[\rho_{2}\right] } & =\left[\rho_{2}\right][\alpha]=\left[\rho_{2}\right] \\
d_{\rho_{1}} & =\sqrt{3}, d_{\rho_{2}}=2, d_{\alpha}=1 .
\end{aligned}
$$

By Proposition 3.3, $[\alpha]$ is lifted to $\boldsymbol{Z}_{2}$ action. By Lemma 3.4, we may assume $\left[\rho_{1}\right][\alpha]=[\alpha]\left[\rho_{1}\right]$.

We consider the following inclusions:

$$
M \supset \rho_{1}(M) \supset \rho_{1}\left(M^{\alpha}\right) .
$$

Let $E \in \mathrm{E}\left(M, \rho_{1}(M)\right)$. By Subsection 2.2, there exists an isometry $v \in M$ such that $E(x)=\rho_{1}\left(v^{*} \bar{\rho}_{1}(x) v\right)$. Since $\rho_{1}\left(M^{\alpha}\right)=\rho_{1}(M)^{\rho_{1}{ }^{\alpha \alpha \rho} \rho_{1}^{-1}}$, there is a unique $\hat{E} \in$ $\mathrm{E}\left(\rho_{1}(M), \rho_{1}\left(M^{\alpha}\right)\right)$ and $\hat{E}$ is given as follows;

$$
\hat{E}(x) \equiv \frac{x+\rho_{1} \circ \alpha \circ \rho_{1}^{-1}(x)}{2} \text { for } x \in \rho_{1}(M) .
$$

We can define $E_{1} \in \mathrm{E}\left(M, \rho_{1}\left(M^{\alpha}\right)\right)$ by

$$
\begin{aligned}
E_{1}(x) & \equiv \hat{E} \circ E(x) \\
& =\frac{E(x)+\rho_{1} \circ \alpha \circ \rho_{1}^{-1}(E(x))}{2} \\
& =\frac{\rho_{1}\left(v^{*}\right) \rho_{1} \bar{\rho}_{1}(x) \rho_{1}(v)+\rho_{1}\left(\alpha\left(v^{*}\right)\right) \rho_{1} \circ \alpha \circ \bar{\rho}_{1}(x) \rho_{1}(\alpha(v))}{2} .
\end{aligned}
$$

Let $\rho_{0} \in \operatorname{End}(M)$ with $\rho_{0}(M)=\rho_{1}\left(M^{\alpha}\right)$. We saw in Subsection 2.2 that $\left[\rho_{0} \bar{\rho}_{0}\right]$ is calculated from $E_{1}$ by using an $M-M$ correspondence and the above relation means that $\left[\rho_{0} \bar{\rho}_{0}\right]$ is contained in

$$
\begin{aligned}
{\left[\rho_{1} \bar{\rho}_{1}\right] \oplus\left[\rho_{1} \circ \alpha \circ \bar{\rho}_{1}\right] } & =\left[\rho_{1} \bar{\rho}_{1}\right] \oplus[\alpha]\left[\rho_{1} \bar{\rho}_{1}\right] \\
& =[i d] \oplus[\alpha] \oplus 2\left[\rho_{2}\right] .
\end{aligned}
$$

Since this contains $[i d]$ with multiplicity one, $\rho_{0}$ is irreducible and $E_{1}$ is the unique expectation. Then

$$
\left[M: \rho_{0}(M)\right]_{0}=\operatorname{Index} E_{1}=(\operatorname{Index} \hat{E})(\operatorname{Index} E)=6 .
$$

By comparing statistical dimension, we have

$$
\left[\rho_{0} \bar{\rho}_{0}\right]=[i d] \oplus[\alpha] \oplus 2\left[\rho_{2}\right] .
$$

Due to $d_{i d}=1, d_{\alpha}=1, d_{\mathrm{P}_{2}}=2, \rho_{0}$ satisfies the condition (v) of Proposition 4.2, 
and a possible decomposition of $\left[\bar{\rho}_{0} \rho_{0}\right]$ is one of the following.

$$
\left[\bar{\rho}_{0} \rho_{0}\right]=[i d] \oplus\left[\beta_{1}\right] \oplus 2\left[\beta_{2}\right] \quad \text { where } \quad d_{\beta_{1}}=1, d_{\beta_{2}}=2,
$$

or

$$
\left[\bar{\rho}_{0} \rho_{0}\right]=\bigoplus_{i}\left[r_{i}\right] \quad \text { where } \quad d_{\gamma_{i}}=1 .
$$

In the second case, the relation between $M$ and $\rho_{0}(M)$ is given by the crossed product by a non-commutative group of order 6 due to Theorem 4.1 and Proposition 4.3 , i.e.

$$
M=\rho_{0}(M) \rtimes S_{3} .
$$

To reject the first possibility, we need the following two lemmas.

Lemma 5.2. Let $\rho \in \operatorname{End}(M)$ with $d_{\rho}=\sqrt{6}$. If $\rho$ is irreducible and $[\rho \bar{\rho}]=$ $[i d] \oplus\left[\alpha_{1}\right] \oplus 2\left[\alpha_{2}\right]$ is the irreducible decomposition such that $d_{\alpha_{1}}=1, d_{\alpha_{2}}=2$, then $\alpha_{1}$ and $\alpha_{2}$ are self-conjugate and the fusion rules are the following.

$$
\begin{aligned}
{\left[\alpha_{1}\right]^{2} } & =[i d], \\
{\left[\alpha_{1} \alpha_{2}\right] } & =\left[\alpha_{2} \alpha_{1}\right]=\left[\alpha_{2}\right], \\
{\left[\alpha_{2}\right]^{2} } & =[i d] \oplus\left[\alpha_{1}\right] \oplus\left[\alpha_{2}\right] .
\end{aligned}
$$

Proof. Self-conjugacy of $\left[\alpha_{1}\right]$ and $\left[\alpha_{2}\right]$ follows from that of $[\rho \bar{\rho}]$. Since $\rho$ satisfies the conditions of Proposition 4.2, $\left[\alpha_{1}\right][\rho]=[\rho]$ and $\left[\alpha_{2}\right][\rho]=2[\rho]$ hold. From the first equality, we have

$$
[\rho \bar{\rho}]=\left[\alpha_{1}\right][\rho \bar{\rho}]=\left[\alpha_{1}\right][i d] \oplus\left[\alpha_{1}\right]\left[\alpha_{1}\right] \oplus 2\left[\alpha_{1}\right]\left[\alpha_{2}\right] .
$$

So, we see that $\left[\alpha_{1}\right]^{2}=[i d]$ and $\left[\alpha_{1}\right]\left[\alpha_{2}\right]=\left[\alpha_{2}\right]$, and by conjugation $\left[\alpha_{2}\right]\left[\alpha_{1}\right]=\left[\alpha_{2}\right]$. From the second equality, we obtain

$$
\begin{aligned}
2[\rho \bar{\rho}] & =\left[\alpha_{2}\right][\rho \bar{\rho}]=\left[\alpha_{2}\right][i d] \oplus\left[\alpha_{2}\right]\left[\alpha_{1}\right] \oplus 2\left[\alpha_{2}\right]\left[\alpha_{2}\right] \\
& =2\left[\alpha_{2}\right] \oplus 2\left[\alpha_{2}\right]^{2} .
\end{aligned}
$$

Therefore, $\left[\alpha_{2}\right]^{2}=[i d] \oplus\left[\alpha_{1}\right] \oplus\left[\alpha_{2}\right]$.

Q.E.D.

Lemma 5.3. If the assumptions of Lemma 5.2 are satisfied, then $[\bar{\rho} \rho]=$ $\bigoplus_{i}\left[r_{i}\right]$ such that $d_{\gamma_{i}}=1$.

Proof. Suppose $[\bar{\rho} \rho]=[i d] \oplus\left[\beta_{1}\right] \oplus 2\left[\beta_{2}\right]$ such that $d_{\beta_{1}}=1, d_{\beta_{2}}=2$. Since the depth of $M \supset \rho(M)$ is two, we define

$$
\begin{aligned}
& V(i d)=1, V\left(\alpha_{1}\right), V\left(\alpha_{2}\right)_{1}, V\left(\alpha_{2}\right)_{2}, \\
& W(i d)=1, W\left(\beta_{1}\right), W\left(\beta_{2}\right)_{1}, W\left(\beta_{2}\right)_{2},
\end{aligned}
$$




$$
\pi_{a b}^{i j\langle\alpha, \beta\rangle,}
$$

etc. as in Section 4. By definition

$$
\begin{aligned}
& \pi\langle i d, i d\rangle=\pi\left\langle\alpha_{1}, i d\right\rangle=\pi\left\langle i d, \beta_{1}\right\rangle=1, \\
& \pi^{\cdot}\left\langle\alpha_{2}, i d\right\rangle=\pi \cdot\left\langle i d, \beta_{2}\right\rangle=\left[\begin{array}{ll}
1 & 0 \\
0 & 1
\end{array}\right] .
\end{aligned}
$$

We may assume $\beta_{1} \circ \bar{\rho}=\bar{\rho}$ and $\alpha_{1} \circ \alpha_{2}=\alpha_{2}$. Then $\alpha_{1}^{2}=\beta_{1}^{2}=i d$ as in the proof of Theorem 4.1. From (5.3.1), (5.3.2), Lemma 5.2 and Proposition 4.5 (iii), we obtain

$$
\begin{aligned}
\pi\left\langle\alpha_{1}, \beta_{1}\right\rangle^{2} & =\pi\left\langle\alpha_{1}, i d\right\rangle=1 \\
\pi^{*}\left\langle\alpha_{2}, \beta_{1}\right\rangle^{2} & =\pi^{*}\left\langle\alpha_{2}, i d\right\rangle=\left[\begin{array}{ll}
1 & 0 \\
0 & 1
\end{array}\right] .
\end{aligned}
$$

By changing the basis $\left\{V\left(\alpha_{2}\right)_{i}\right\}_{i}$, we may assume that $\pi^{\circ}\left\langle\alpha_{2}, \beta_{1}\right\rangle$ is diagonal.

First, we show $\pi\left\langle\alpha_{1}, \beta_{1}\right\rangle=-1$. If $\pi\left\langle\alpha_{1}, \beta_{1}\right\rangle=+1$, from Proposition 4.5 (iv) and Lemma 5.2 we obtain

$$
\pi^{t r}\left\langle\alpha_{2}, \beta_{1}\right\rangle^{2}=\pi\left\langle i d, \beta_{1}\right\rangle+\pi\left\langle\alpha_{1}, \beta_{1}\right\rangle+\pi^{t r}\left\langle\alpha_{2}, \beta_{1}\right\rangle .
$$

From (5.3.1) and this, we also obtain

$$
\pi^{t r}\left\langle\alpha_{2}, \beta_{1}\right\rangle^{2}=2+\pi^{t r}\left\langle\alpha_{2}, \beta_{1}\right\rangle \text {. }
$$

So, $\pi^{t r}\left\langle\alpha_{2}, \beta_{1}\right\rangle=-1$ or 2 . Since $\pi^{\circ}\left\langle\alpha_{2}, \beta_{1}\right\rangle$ is diagonal and unitary, this and (5.3.4) imply

$$
\pi^{\cdot}\left\langle\alpha_{2}, \beta_{1}\right\rangle=\left[\begin{array}{ll}
1 & 0 \\
0 & 1
\end{array}\right]
$$

This and $\pi\left\langle\alpha_{1}, \beta_{1}\right\rangle=+1$ mean

$$
\beta_{1}\left(V\left(\alpha_{1}\right)\right)=V\left(\alpha_{1}\right), \beta_{1}\left(V\left(\alpha_{2}\right)_{i}\right)=V\left(\alpha_{2}\right)_{i} .
$$

We have also taken $\beta_{1}$ out of $\left[\beta_{1}\right]$ such that $\beta_{1} \circ \bar{\rho}=\bar{\rho}$. Since $\bar{\rho}(M)$ and $\left\{V\left(\alpha_{j}\right)_{i}\right\}_{i j}$ generate $M$ by Proposition 4.5 (vi), we obtain $\beta_{1}=i d$. This is impossible and we conclude $\pi\left\langle\alpha_{1}, \beta_{1}\right\rangle=-1$.

Second, we show

$$
\pi_{i r}^{\cdot}\left\langle\alpha_{2}, \beta_{2}\right\rangle=\left[\begin{array}{rr}
-1 & 0 \\
0 & 0
\end{array}\right]
$$

From eq. (5.3.5), we now have $\pi^{t r}\left\langle\alpha_{2}, \beta_{1}\right\rangle^{2}=\pi^{t r}\left\langle\alpha_{2}, \beta_{1}\right\rangle$ and so $\pi^{t r}\left\langle\alpha_{2}, \beta_{1}\right\rangle=0$ 
or 1. Since $\pi^{\circ}\left\langle\alpha_{2}, \beta_{1}\right\rangle$ is unitary and diagonal, this and (5.3.4) imply $\pi^{t r}\left\langle\alpha_{2}, \beta_{1}\right\rangle$ $=0$. We may assume

$$
\pi^{\circ}\left\langle\alpha_{2}, \beta_{1}\right\rangle=\left[\begin{array}{rr}
1 & 0 \\
0 & -1
\end{array}\right] .
$$

By Proposition $4.5(\mathrm{v})$ and $\left[\beta_{2}\right]\left[\beta_{1}\right]=\left[\beta_{1}\right]\left[\beta_{2}\right]=\left[\beta_{2}\right]$,

$$
\pi_{i r}^{\circ}\left\langle\alpha_{2}, \beta_{2}\right\rangle \pi^{\circ}\left\langle\alpha_{2}, \beta_{1}\right\rangle=\pi^{\circ}\left\langle\alpha_{2}, \beta_{1}\right\rangle \pi_{i r}^{\circ}\left\langle\alpha_{2}, \beta_{2}\right\rangle=\pi_{i r}^{\circ}\left\langle\alpha_{2}, \beta_{2}\right\rangle .
$$

Therefore,

$$
\pi_{i r}^{\circ}\left\langle\alpha_{2}, \beta_{2}\right\rangle=\left[\begin{array}{ll}
a & 0 \\
0 & 0
\end{array}\right] \quad a \in \mathbb{C} .
$$

Also, the relation $\left[\beta_{2}\right]^{2}=[i d] \oplus\left[\beta_{1}\right] \oplus\left[\beta_{2}\right]$ implies

$$
\begin{aligned}
\pi_{i r}^{\circ}\left\langle\alpha_{2}, \beta_{2}\right\rangle^{2} & =\left[\begin{array}{ll}
1 & 0 \\
0 & 1
\end{array}\right]+\left[\begin{array}{rr}
1 & 0 \\
0 & -1
\end{array}\right]+\pi_{i r}^{\circ}\left\langle\alpha_{2}, \beta_{2}\right\rangle \\
& =\left[\begin{array}{ll}
2 & 0 \\
0 & 0
\end{array}\right]+\pi_{i r}^{\circ}\left\langle\alpha_{2}, \beta_{2}\right\rangle .
\end{aligned}
$$

So $a=2$ or -1 .

In the same way as $\pi^{\circ}\left\langle\alpha_{2}, \beta_{1}\right\rangle$, we obtain

$$
\pi_{\circ}\left\langle\alpha_{1}, \beta_{2}\right\rangle^{2}=\left[\begin{array}{ll}
1 & 0 \\
0 & 1
\end{array}\right] .
$$

By changing basis $\left\{W\left(\beta_{2}\right)_{a}\right\}$, we may assume

$$
\begin{aligned}
& \pi_{\circ}\left\langle\alpha_{1}, \beta_{2}\right\rangle=\left[\begin{array}{rr}
1 & 0 \\
0 & -1
\end{array}\right], \\
& \pi_{\circ}^{t r}\left\langle\alpha_{2}, \beta_{2}\right\rangle=\left[\begin{array}{ll}
b & 0 \\
0 & 0
\end{array}\right],
\end{aligned}
$$

where $b=2$ or -1 , as before. Since $\pi_{0}^{\circ}\left\langle\alpha_{2}, \beta_{2}\right\rangle$ is unitary, if $a$ or $b$ is 2 , then

$$
\pi_{\circ}^{\circ}\left\langle\alpha_{2}, \beta_{2}\right\rangle=\left[\begin{array}{rrrr}
1 & 0 & 0 & 0 \\
0 & 1 & 0 & 0 \\
0 & 0 & 1 & 0 \\
0 & 0 & 0 & -1
\end{array}\right] .
$$

Since $\bar{\rho}(M)$ and $\left\{V\left(\alpha_{j}\right)_{i}\right\}_{i j}$ generate $M$, this and eq. (5.3.8) mean

$$
W\left(\beta_{2}\right)_{1} W\left(\beta_{2}\right)_{1}^{*}, W\left(\beta_{2}\right)_{2} W\left(\beta_{2}\right)_{2}^{*} \in M \cap \beta_{2}(M)^{\prime} .
$$


This is impossible because $\beta_{2}$ is irreducible. Therefore we obtain (5.3.6). By the above argument, we may aslo assume

$$
\begin{aligned}
\pi_{\bullet}\left\langle\alpha_{1}, \beta_{2}\right\rangle & =\left[\begin{array}{rr}
1 & 0 \\
0 & -1
\end{array}\right], \\
\pi_{\bullet}^{t r}\left\langle\alpha_{2}, \beta_{2}\right\rangle & =\left[\begin{array}{rr}
-1 & 0 \\
0 & 0
\end{array}\right] .
\end{aligned}
$$

But we do not make this assumption because we will make a further change of the basis $\left\{W\left(\beta_{2}\right)_{a}\right\}_{a=1,2}$. Instead of this assumption, we use the information that $\pi_{\cdot}^{t r}\left\langle\alpha_{2}, \beta_{2}\right\rangle$ is Hermitian, $\operatorname{Sp}_{\cdot}^{t r}\left\langle\alpha_{2}, \beta_{2}\right\rangle=\{0,-1\}$ and $\pi_{t r}\left\langle\alpha_{1}, \beta_{2}\right\rangle=0$.

Finally we derive a contradiction. $\operatorname{By}\left[\beta_{1}\right]\left[\beta_{2}\right]=\left[\beta_{2}\right]$ and Proposition 4.5. (iii), we obtain $\sum_{k} \pi_{\bullet}^{i k}\left\langle\alpha_{2}, \beta_{1}\right\rangle \otimes \pi_{\bullet}^{k j}\left\langle\alpha_{2}, \beta_{2}\right\rangle=\sum_{k} \pi^{i k}\left\langle\alpha_{2}, \beta_{1}\right\rangle \pi_{\bullet}^{k j}\left\langle\alpha_{2}, \beta_{2}\right\rangle$ is unitarily equivalent to $\pi_{\cdot}^{i j}\left\langle\alpha_{2}, \beta_{2}\right\rangle$. Due to (5.3.7), this means that there exists a unitary $U \in \mathrm{M}(2)$ such that

$$
\begin{aligned}
\pi_{\bullet}^{11}\left\langle\alpha_{2}, \beta_{2}\right\rangle U & =U \pi_{\bullet}^{11}\left\langle\alpha_{2}, \beta_{2}\right\rangle, \\
\pi_{\bullet}^{12}\left\langle\alpha_{2}, \beta_{2}\right\rangle U & =U \pi_{\bullet}^{12}\left\langle\alpha_{2}, \beta_{2}\right\rangle, \\
-\pi_{\bullet}^{21}\left\langle\alpha_{2}, \beta_{2}\right\rangle U & =U \pi_{\bullet}^{21}\left\langle\alpha_{2}, \beta_{2}\right\rangle, \\
-\pi_{\bullet}^{22}\left\langle\alpha_{2}, \beta_{2}\right\rangle U & =U \pi_{\bullet}^{22}\left\langle\alpha_{2}, \beta_{2}\right\rangle .
\end{aligned}
$$

By changing the basis $\left\{W\left(\beta_{2}\right)_{a}\right\}_{a=1,2}$, we may assume

$$
U=\left[\begin{array}{cc}
\omega_{1} & 0 \\
0 & \omega_{2}
\end{array}\right] \quad \omega_{1} \neq \omega_{2},
$$

(if $\omega_{1}=\omega_{2}, \pi^{21}\left\langle\alpha_{2}, \beta_{2}\right\rangle=\pi_{.}^{22}\left\langle\alpha_{2}, \beta_{2}\right\rangle=0$ and $\pi_{\bullet}^{\cdot}\left\langle\alpha_{2}, \beta_{2}\right\rangle$ can not be unitary.) and so

$$
\begin{aligned}
\pi_{\bullet}^{11}\left\langle\alpha_{2}, \beta_{2}\right\rangle & =\left[\begin{array}{ll}
a_{1} & 0 \\
0 & a_{2}
\end{array}\right], \\
\pi_{\bullet}^{12}\left\langle\alpha_{2}, \beta_{2}\right\rangle & =\left[\begin{array}{ll}
b_{1} & 0 \\
0 & b_{2}
\end{array}\right], \\
\pi_{\bullet}^{21}\left\langle\alpha_{2}, \beta_{2}\right\rangle & =\left[\begin{array}{ll}
0 & c_{1} \\
c_{2} & 0
\end{array}\right], \\
\pi_{\bullet}^{22}\left\langle\alpha_{2}, \beta_{2}\right\rangle & =\left[\begin{array}{cc}
0 & d_{1} \\
d_{2} & 0
\end{array}\right], \\
\pi_{\bullet}^{\cdot}\left\langle\alpha_{2}, \beta_{2}\right\rangle & =\left[\begin{array}{cccc}
a_{1} & 0 & b_{1} & 0 \\
0 & a_{2} & 0 & b_{2} \\
0 & c_{1} & 0 & d_{1} \\
c_{2} & 0 & d_{2} & 0
\end{array}\right],
\end{aligned}
$$




$$
\begin{aligned}
& \pi_{i r}^{\circ}\left\langle\alpha_{2}, \beta_{2}\right\rangle=\left[\begin{array}{cc}
a_{1}+a_{2} & b_{1}+b_{2} \\
0 & 0
\end{array}\right], \\
& \pi_{\bullet}^{t r}\left\langle\alpha_{2}, \beta_{2}\right\rangle=\left[\begin{array}{ll}
a_{1} & d_{1} \\
d_{2} & a_{2}
\end{array}\right] .
\end{aligned}
$$

From (5.3.6), we obtain $a_{1}+a_{2}=-1$ and $b_{1}+b_{2}=0$. Since $\pi_{\circ}^{8 r}\left\langle\alpha_{2}, \beta_{2}\right\rangle$ is Hermitian and $\mathrm{Sp} \pi_{\circ}^{t r}\left\langle\alpha_{2}, \beta_{2}\right\rangle=\{0,1\}$, we obtain

$$
a_{1}, a_{2} \in \mathbb{R}, \quad d_{1}=\bar{d}_{2}, \quad a_{1} a_{2}-\left|d_{1}\right|^{2}=0 .
$$

We write as follows.

$$
\begin{gathered}
\pi_{\circ}^{\circ}\left\langle\alpha_{2}, \beta_{2}\right\rangle=\left[\begin{array}{rrrr}
a_{1} & 0 & b & 0 \\
0 & a_{2} & 0 & -b \\
0 & c_{1} & 0 & d \\
c_{2} & 0 & \bar{d} & 0
\end{array}\right], \\
a_{1}, a_{2} \in \mathbb{R}, a_{1} a_{2}=|d|^{2}, a_{1}+a_{2}=-1 .
\end{gathered}
$$

From $\left[\beta_{2}\right]^{2}=[i d] \oplus\left[\beta_{1}\right] \oplus\left[\beta_{2}\right]$ and Proposition 4.5 (iii), we obtain that

$$
\sum_{k=1}^{2} \pi_{\bullet}^{i k}\left\langle\alpha_{2}, \beta_{2}\right\rangle \otimes \pi_{\bullet}^{k j}\left\langle\alpha_{2}, \beta_{2}\right\rangle
$$

is unitarily equivalent to

$$
\pi^{i j}\left\langle\alpha_{2}, i d\right\rangle \oplus \pi^{i j}\left\langle\alpha_{2}, \beta_{1}\right\rangle \oplus \pi_{0}^{i j}\left\langle\alpha_{2}, \beta_{2}\right\rangle .
$$

For $i=1, j=2$

$$
\pi_{\circ}^{11}\left\langle\alpha_{2}, \beta_{2}\right\rangle \otimes \pi_{\circ}^{12}\left\langle\alpha_{2}, \beta_{2}\right\rangle+\pi_{\circ}^{12}\left\langle\alpha_{2}, \beta_{2}\right\rangle \otimes \pi_{\circ}^{22}\left\langle\alpha_{2}, \beta_{2}\right\rangle \simeq 0 \oplus 0 \oplus \pi_{\circ}^{12}\left\langle\alpha_{2}, \beta_{2}\right\rangle .
$$

This means

$$
\left[\begin{array}{rr}
a_{1} & 0 \\
0 & a_{2}
\end{array}\right] \otimes\left[\begin{array}{rr}
b & 0 \\
0 & -b
\end{array}\right]+\left[\begin{array}{rr}
b & 0 \\
0 & -b
\end{array}\right] \otimes\left[\begin{array}{ll}
0 & d \\
\bar{d} & 0
\end{array}\right] \simeq\left[\begin{array}{rrrr}
0 & 0 & 0 & 0 \\
0 & 0 & 0 & 0 \\
0 & 0 & b & 0 \\
0 & 0 & 0 & -b
\end{array}\right]
$$

If $b \neq 0$, we divide by $b$ and obtain

$$
\left[\begin{array}{rrrr}
a_{1} & d & 0 & 0 \\
d & -a_{1} & 0 & 0 \\
0 & 0 & a_{2} & -d \\
0 & 0 & -d & -a_{2}
\end{array}\right] \simeq\left[\begin{array}{rrrr}
0 & 0 & 0 & 0 \\
0 & 0 & 0 & 0 \\
0 & 0 & 1 & 0 \\
0 & 0 & 0 & -1
\end{array}\right]
$$

Due to $a_{1} a_{2}=|d|^{2}, a_{1}+a_{2}=-1$, 


$$
\begin{aligned}
& \operatorname{Sp}\left[\begin{array}{rrrr}
a_{1} & d & 0 & 0 \\
d & -a_{1} & 0 & 0 \\
0 & 0 & a_{2} & -d \\
0 & 0 & -d & -a_{2}
\end{array}\right]=\left\{ \pm \sqrt{a_{1}^{2}+|d|^{2}}, \pm \sqrt{a_{2}^{2}+|d|^{2}}\right\} \\
& =\left\{ \pm \sqrt{-a_{1}}, \pm \sqrt{-a_{2}}\right\} .
\end{aligned}
$$

So $a_{1}$ or $a_{2}=0$, and $d=0$. But this is impossible because $\pi_{:}^{*}\left\langle\alpha_{2}, \beta_{2}\right\rangle$ must be unitary and $a_{1}+a_{2}=-1$. We are now left with the alternative $b=0$. But this is also impossible because, $b=0$ implies $\left|a_{1}\right|=\left|a_{2}\right|=1$ due to the unitarity of $\pi^{\circ}\left\langle\alpha_{2}, \beta_{2}\right\rangle$, while $a_{1}$ and $a_{2}$ are real and $a_{1}+a_{2}=-1$. Thus we reached a contradiction.

Q.E.D.

Completion of proof of Theorem 5.1. By Lemma 5.3, we obtain $\left[\bar{\rho}_{0}\right]\left[\rho_{0}\right]$ $=\bigoplus_{g \in S_{3}}\left[\gamma_{g}\right]$. By Theorem 4.1 and the remark after Proposition 4.2, we obtain

$$
M=\rho_{0}(M) \underset{\rho_{0}^{\circ} \gamma^{\circ} \rho_{0}^{-1}}{\rtimes} S_{3}
$$

Since $\left[\rho_{1}(M): \rho_{0}(M)\right]=2$, there exists a $Z_{2}$ action $\theta$ on $M$ such that

$$
\rho_{1}(M)=\rho_{0}(M) \underset{\rho_{0} \circ \theta \circ \rho_{0}^{-1}}{\searrow} Z_{2}
$$

By the definition of crossed product, there exists a unitary $u \in M$ such that

$$
\rho_{0} \circ \theta(x)=\rho_{0} \circ \theta \circ \rho_{0}^{-1}\left(\rho_{0}(x)\right)=u \rho_{0}(x) u^{*} .
$$

This means $\left[\rho_{0} \circ \theta\right]=\left[\rho_{0}\right]$ and by conjugation, $[\theta]\left[\bar{\rho}_{0}\right]=\left[\bar{\rho}_{0}\right]$. Multiplying both sides of the equality $\left[\bar{\rho}_{0} \rho_{0}\right]=\oplus\left[\gamma_{g}\right]$ by $[\theta]$ from left, we have

$$
\left[\bar{\rho}_{0} \rho_{0}\right]=[\theta]\left[\bar{\rho}_{0} \rho_{0}\right]=\oplus[\theta]\left[r_{g}\right] \text {. }
$$

Therefore $[\theta] \in\left\{\left[r_{g}\right]\right\}$ i.e. $\{i d, \theta\}$ is cocycle conjugate to a subgroup of $\left\{r_{g}\right\}$. Then

$$
M \supset N \simeq \rho_{0}(M) \rtimes S_{3} \supset \rho_{0}(M) \rtimes S_{2} .
$$

From the classification of group actions on the type $\mathrm{III}_{1}$ AFD factor [KST, Theorem 20], we obtain the desired conclusion of Theorem 5.1. Q.E.D.

Corollary 5.4. Let $M \supset N$ be a pair of factors with $[M: N]_{0}=6$ and $M \cap$ $N^{\prime}=C$. If the depth of $M \supset N$ is two, the relation between $M$ and $N$ is one of the following.

(i) There exist a group $G$ and its outer action on $M$ such that $N=M^{G}$.

(ii) There exist a group $G$ and its outer action on $N$ such that $M=N \rtimes G$. 
Proof. Suppose the contrary. Due to Lemma 3.8, we may assume that $M$ and $N$ are properly infinite and $M$ is isomorphic to $N$, because the principal graph does not change after taking tensor product. (Note that the above two conditions are characterized by the principal graph of $M \supset N$ or the principal graph of $M_{1} \subset M$, where $M_{1}$ is the extension of $M$ by $N$ [K3] [PP1, Corollary 1.1.6].) Let $\rho \in \operatorname{End}(M)$ with $N=\rho(M)$. Thanks to Theorem 4.1 and Proposition $4.2,[\rho]$ and $[\bar{\rho}]$ must satisfy the condition of Lemma 5.2. But this is impossible because of Lemma 5.3.

\section{§。. Comments}

6.1. In the theory of Ocneanu's string algebra construction, there is no criterion for the distinguished point in the case of general graphs. (In the case of Coxeter graphs, the following (i) was announced by A. Ocneanu [01, page 161-162].) By the arguments of Subsection 2.3, we have the following criterions for the distinguished point and the existence of a flat connection.

Theorem 6.1. (i) The distinguished point must be the point with the minimal component of the Perron-Forbenius eigenvector.

(ii) If we normalize the Perron-Frobenius eigenvector such that the minimal component is one, all components must belong to $\left\{2 \cos \frac{\pi}{N} ; N=3,4, \cdots\right\} \cup$ $[2, \infty)$

These criterions essentially follow from the local index formula in [PP2, Corollary 3.2]. $\quad D_{5}, E_{7}$ and the two graphs of U. Haagerup and J.K. Schou [HS] shown in Fig. 17 do not have a point satisfying these criterions and hence can not appear as a principal graph.
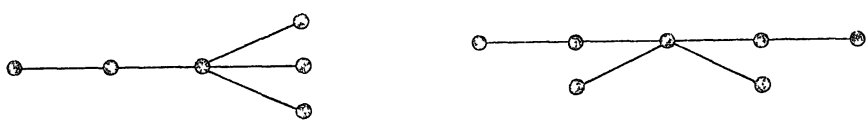

Fig. 17. Two graphs in the list of [HS].

6.2. As we saw in Section 3 and just above, the properties of the statistical dimensions such as the restriction of the values, additivity, and multiplicativity, are very powerful for rejecting non-flat graphs. But we can prove Theorem 3.7 solely on the basis of algebraic calculation of fusion rules. We omit the detail.

6.3. In [Ka] $Y$. Kawahigashi proves the non-existence of $D_{\text {odd }}$ and $E_{7}$ para- 
groups and the existence of $D_{\text {even }}$ paragroups by the argument about flatness of graphs. He also shows the numerical evidence for flatness of $E_{6}$ and $E_{8}$. His method is constructive and this is the strong point of his method. But it seems very hard to show flatness for general graphs.

The existence of a flat connection of a graph implies indireclty the existence of consistent fusion rules associated with the graph, through the theory of operator algebras. But Lemma 5.3 shows that the converse is not true, i.e. the consistency of the formal calcuration of fusion rules does not necessarily imply the existence of a flat connection. The graph in Fig. 18 is also a counter example. Indeed, suppose the principal graph of $M \supset \rho(M)$ is as in Fig. 18. Then we can take $\rho_{1}$ such that

$$
\rho_{1}(M)=\rho(M), \quad\left[\rho_{1}\right]^{2}=[i d] \oplus\left[\rho_{1}\right] \oplus\left[\rho_{1}\right],
$$

as in the case of $A_{2 n}$. This implies that we can construct $\rho_{1}$ using the Cuntz algebra $O_{3}[\mathrm{I}]$. But direct computation shows that this is impossible.

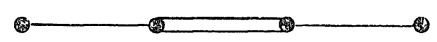

Fig. 18. An example of a non-flat graph which has consistent fusion rules.

We must clarify the relation between flat connections and fusion rules to simplify the axiom of flatness.

6.4. It is not hard to calculate fusion rules for Coxeter graphs with the PerronFrobenius eigenvalue two such as $D_{n}^{(1)}, E_{6}^{(1)}, E_{7}^{(1)}$ and $E_{8}^{(1)} . \quad\left(\mathrm{A}_{n}^{(1)}\right.$ is not interesting because the corresponding sector is reducible and is a sum of two automorphisms.) The method in Section 5 is also applicable to the case of $D_{6}^{(1)}$ and $E_{6}^{(1)}$. But more complicated calculation is needed for the step corresponding to Lemma 5.3.

6.5. In the theory of the $\mathrm{II}_{1}$ AFD factor, the counterpart of the notion of the canonical endomorphism is the canonical shift, and that of self-conjugate endomorphism is Choda's roots of the canonical shift [C]. She shows in [C] the following fact.

Fact 6.2. Let $R \supset P$ be a pair of $I I_{1}$ factors. If $[R: P]<4$ or $P$ is the fixed point algebra under an outer action of a finite abelian group, then there exists $\rho \in \operatorname{End}(R)$ such that $\rho(R)=P$ and $\rho^{2}$ is the canonical shift.

In [I], for every finite abelian group $G$ of $\operatorname{order} n$, we construct self-conjugate endomorphism on type $\mathrm{II}_{1 / n} \mathrm{AFD}$ factor, whose image is the fixed point 
algebra under an outer action of $G$, by using Cuntz algebra $O_{n}$. Since an outer action of any finite abelian group on the type $\mathrm{III}_{1} \mathrm{AFD}$ factor is unique [KST, Theorem 20], by taking tensor product with the type $\mathbb{I I I}_{1}$ factor, we have the following fact.

Fact 6.3. Let $M$ be the type $\mathbb{I I}_{1}$ AFD factor and $N$ be the fixed point algebra of $M$ under the outer action of a finite abelian group. Then there exists a self-conjugate $\rho \in \mathbb{E n d}(M)$ such that $\rho(M)=N$.

In analogy with the case of the $\mathbb{I}_{1} \mathrm{AFD}$ factor we make the folloiwng conjecture.

Conjecture 6.4. Let $M \supset N$ be a pair of type III 1 ASD factors. If $[M: N]$ $<4$, then there exists a self-conjugate $\rho \in \operatorname{End}(M)$ such that $\rho(M)=N$

In the case that the principal graph of $M \supset N$ is $A_{\text {even, }}$ we saw in Section 3 that this conjecture is true.

\section{§7. Appendix。 In the Case of Type HI $_{\infty}$ Factors}

Proposition \%.1。 Let $M \supset N$ be a pair of a type $I I_{\infty}$ factors with $[M: N]_{0}<$ $\infty$. Then every non-zero projection in $M \cap N^{\prime}$ is infinite.

Proof. Let $\tau_{1}, \tau_{2}$ be the canonical traces on $M$ and $N$, and let $E$ be the minimal expectation from $M$ to $N$. We define a faithful nomral semi-finite weight by $\tau_{2} \circ E$. Then there exists a unique positive self-adjoint operator $h$ affiliated with $M$ such that

$$
\tau_{2} \circ E=\tau_{1}(h \cdot)
$$

Due to $\sigma_{\mid N}^{\tau_{2^{\circ} E}}=\sigma^{\tau}=i d_{N}, \sigma^{E}=i d_{M \cup N^{\prime}}$ and $\sigma_{t}^{\tau_{2}{ }^{\circ} E}=\mathrm{Ad} h^{i t}, h$ is affiliated with $Z(M \cap$ $\left.N^{\prime}\right)$, and in fact $h$ belongs to $Z\left(M \cap N^{\prime}\right)$ because of $\operatorname{dim} M \cap N^{\prime}<\infty$. Let $p$ be a minimal projection in $M \cap N^{\prime}$. Then $h p=c p$ for some scalar $c>0$. So

$$
\tau_{1}(p)=\frac{1}{c} \tau_{1}(h p)=\frac{1}{c} \tau_{2}(E(p)) .
$$

Since $E(p)$ is a positive scalar we obtain $\tau_{1}(p)=\infty$.

Q.E.D.

\section{Ackmowledgments}

The author would like to thank $\mathrm{H}$. Araki for introducing him to the theory of operator algebras and discussions. He is grateful to M. Choda, Y. Kawahigashi and in particular $\mathbb{H}$. Kosaki for stimulating discussions. $\mathrm{He}$ is also 
grateful to I. Ojima for introducing him to the theory of superselection sectors and R. Longo for suggesting him Lemma 3.8.

\section{References}

[C] Connes, A., Spatial theory of von Neumann algebra, J. Funct. Anla.35 (1980), 153164.

[Ch] Choda, M., in preparation.

[DHR] Doplicher, S., Haag, R. and Roberts, J.E., Local observables and particle statisties I, Commun. Math. Phys., 23 (1971), 199-230.

[GHJ] Goodman, F., de la Harpe, P. and Jones, V., Coxeter graphs and towers of algebras, MSRI Publications 14, Springer Verlag, 1989.

[H] Hiai, F., Minimizing indices of conditional expectations on a subfactor, Publ. RIMS, Kyoto Univ., 24 (1988), 673-678.

[Ha] Haggerup, U., Operator valued weights in von Neumann algebras I, II, J. Funct. Anal. 32 (1979), 175-206; 33 (1979), 339-361.

[HS] Haagerup, U. and Schou, J.K., preprint.

[I] Izumi, M., in preparation.

[J] Jones, V., Index for subfactors, Invent. Math., 72 (1983), 1-25.

[Ka] Kawahigashi, Y., On flatness of Ocneanu's connection on the Dynkin diagrams and classification of subfactors, preprint.

[KST] Kawahigashi, Y., Sutherland, C.E. and Takesaki, M., The structure of an injective factor and the cocycle conjugacy of discrete abelian group action, to appear in Acta. Math.

[K1] Kosaki, H., Extension of Jones theory on index to arbitrary factors, J. Funct. Anal., 66 (1986), 123-140.

[K2] - private communication.

[K3] - Charaeterization of crossed prodnct (properly infinite case), Pacific $J$. Math., 137 (1989), 159-167.

[KL] Kosaki, H. and Longo, R., A remark on the minimal index of subfactors, preprint.

[L1] Longo, R., Index of subfactors and statistics of quantum fields. I, Commun. Math. Phys., 126 (1989), 217-247.

[L2] - Index of subfactors and statistics of quantum fields. II, Commun. Math. Phys., 130 (1990), 285-309.

[L3] — Simple injective subfactors, Adv. Math., 63 (1987), 152-171.

[L4] —, Minimal index and braided subfactors, preprint.

[Li] Loi, P.H., Sur la théorie de l'indice et les fateurs de type III, C.R. Acad. Sci. Paris, 305 (1987), 423-426.

[01] Ocneanu, A., Quantized group string algebra and Galois theory for algebra, in Operator algebras and applications, 2 (Warwick, 1987), London Math. Snc. Lect. Note Series 136, Cambridge University Press, (1988), 119-172.

[02] - Quantum symmetry, differential geometry of finite graphs and classification of subfactors, University of Tokyo Seminary Notes, (Notes recorded by Y. Kawahigashi), 1990.

[P1] Popa, S., Classification of subfactors: reduction to commuting squares, Invent. Math., 181 (1990), 19-43.

[P2] — Sur la classification des suusfacteurs d'indice fini du facteur hyperfini, C. R Acad. Sc. Paris, 311 (1990), 95-100.

[P3] - Correspondences, preprint.

[PP1] Pimsner, M. and Popa, S., Finite dimensional approximation of algebras and ob- 
struction for the index, prepeint.

[PP2] Pimsner, M. and Popa, S., Iterating the basic construction, Trans. Amer. Math. Soc. 310 (1988), 127-133.

[W1] Wenzl, H., Heck algebras of type $A_{n}$ and subfactors, Invent. Math., 92 (1988), 349383.

[W2] — Lecture at the Mittag-Leffler Institute, 1988.

Note added in proof: After submitting this paper the author received a preprint of V.S. Sunder and A.K. Vijayarajan "On the non-occurrence of the Coxeter graphs $E_{7}$ and $D_{2 n+1}$ as the principal graph of an inclusion of $I I_{1}$ factors" on the non-existence of $D_{\text {odd }}$ and $E_{7}$ paragroups. 